OPEN ACCESS

Edited by:

Péter Poór,

University of Szeged, Hungary

Reviewed by:

Seongbin Hwang,

Sejong University, South Korea Oswaldo Valdes-Lopez,

National Autonomous University

of Mexico, Mexico

*Correspondence:

Shanshan Liu

ars336699@aliyun.com

Pengfei $X u$

xupengfei@neau.edu.cn

Shuzhen Zhang

zhangshuzhen@neau.edu.cn

${ }^{\dagger}$ These authors have contributed equally to this work

Specialty section:

This article was submitted to Plant Pathogen Interactions,

a section of the journal

Frontiers in Plant Science

Received: 20 November 2019 Accepted: 04 January 2021 Published: 28 January 2021

Citation:

Liu T, Wang H, Liu Z, Pang Z, Zhang $C$, Zhao $M$, Ning $B$, Song $B$, Liu S, He Z, Wei W, Wu J, Liu Y, Xu P

and Zhang $S$ (2021) The $26 S$

Proteasome Regulatory Subunit GmPSMD Promotes Resistance to Phytophthora sojae in Soybean.

Front. Plant Sci. 12:513388.

doi: $10.3389 / \mathrm{fp} / \mathrm{s} .2021 .513388$

\section{The 26S Proteasome Regulatory Subunit GmPSMD Promotes Resistance to Phytophthora sojae in Soybean}

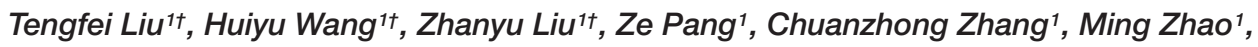
Bin Ning ${ }^{1}$, Bo Song ${ }^{1}$, Shanshan Liu ${ }^{1 *}$, Zili He ${ }^{1}$, Wanling Wei ${ }^{1}$, Junjiang $W^{2}{ }^{2}$, Yaguang Liu' ${ }^{1}$ Pengfei $\mathrm{Xu}^{1 *}$ and Shuzhen Zhang ${ }^{1 *}$

' Key Laboratory of Soybean Biology of Chinese Education Ministry, Soybean Research Institute, Northeast Agricultural University, Harbin, China, ${ }^{2}$ Key Laboratory of Soybean Cultivation of Ministry of Agriculture P. R. China, Soybean Research Institute of Heilongjiang Academy of Agricultural Sciences, Harbin, China

Phytophthora root rot, caused by Phytophthora sojae is a destructive disease of soybean (Glycine max) worldwide. We previously confirmed that the bHLH transcription factor GmPIB1 ( $P$. sojae-inducible bHLH transcription factor) reduces accumulation of reactive oxygen species $(R O S)$ in cells by inhibiting expression of the peroxidase-related gene GmSPOD thus improving the resistance of hairy roots to $P$. sojae. To identify proteins interacting with GmPIB1 and assess their participation in the defense response to $P$. sojae, we obtained transgenic soybean hairy roots overexpressing GmPIB1 by Agrobacterium rhizogenes mediated transformation and examined GmPIB1 proteinprotein interactions using immunoprecipitation combined with mass spectrometry. We identified 392 proteins likely interacting with GmPIB1 and selected 20 candidate genes, and only 26S proteasome regulatory subunit GmPSMD (Genbank accession no. XP_014631720) interacted with GmPIB1 in luciferase complementation and pull-down experiments and yeast two-hybrid assays. Overexpression of GMPSMD (GMPSMD-OE) in soybean hairy roots remarkably improved resistance to $P$. sojae and RNA interference of GmPSMD (GmPSMD -RNAi) increased susceptibility. In addition, accumulation of total ROS and hydrogen peroxide $\left(\mathrm{H}_{2} \mathrm{O}_{2}\right)$ in GMPSMD-OE transgenic soybean hairy roots were remarkably lower than those of the control after $P$. sojae infection. Moreover, in GmPSMD-RNAi transgenic soybean hairy roots, $\mathrm{H}_{2} \mathrm{O}_{2}$ and the accumulation of total ROS exceeded those of the control. There was no obvious difference in superoxide anion $\left(\mathrm{O}_{2}{ }^{-}\right)$content between control and transgenic hairy roots. Antioxidant enzymes include peroxidase (POD), glutathione peroxidase (GPX), superoxide dismutase (SOD), catalase (CAT) are responsible for ROS scavenging in soybean. The activities of these antioxidant enzymes were remarkably higher in GmPSMD-OE transgenic soybean hairy roots than those in control, but were reduced in GmPSMD-RNAi transgenic soybean hairy roots. Moreover, the activity of $26 \mathrm{~S}$ proteasome in GmPSMD-OE and GmPIB1OE transgenic soybean hairy roots was significantly higher than that in control and was significantly lower in PSMD-RNAi soybean hairy roots after $P$. sojae infection. These data 
suggest that GMPSMD might reduce the production of ROS by improving the activity of antioxidant enzymes such as POD, SOD, GPX, CAT, and GmPSMD plays a significant role in the response of soybean to $P$. sojae. Our study reveals a valuable mechanism for regulation of the pathogen response by the $26 \mathrm{~S}$ proteasome in soybean.

Keywords: soybean, Phytophthora sojae, GmPIB1, GmPSMD, ROS

\section{INTRODUCTION}

Phytophthora root rot is caused by the oomycete pathogen Phytophthora sojae Kaufmann and Gerdemann and destroys soybean crops worldwide (Schmitthenner, 1972, 1985; Tyler, 2007). Breeding resistant cultivars is an effective and economical measure to control this disease; however, rapid changes in the pathogen population quickly overcome the resistance of new cultivars (Schmitthenner et al., 1996). Therefore, isolating resistance-related genes and studying resistance mechanisms has great potential value for improving soybean disease resistance through genetic engineering.

Protein homeostasis facilitates cell senescence and protects cells from disease. As the main cellular protease complex, the $26 \mathrm{~S}$ proteasome is at the core of maintaining protein homeostasis in cells (Smalle and Vierstra, 2004; Kurepa and Smalle, 2008). The $26 \mathrm{~S}$ proteasome can be divided into two subcomplexes: the core particle (20S) and the regulatory particle (19S). The 19S regulatory particle consists of at least 19 proteins and the $20 \mathrm{~S}$ core particle consists of 28 proteins. The difference between $20 \mathrm{~S}$ core particles and $19 \mathrm{~S}$ regulatory particles is that $20 \mathrm{~S}$ core particles can degrade proteins without hydrolyzing ATP (KishTrier and Hill, 2013), and they cannot recognize and degrade protein substrates labeled by ubiquitin chains (Stadtmueller et al., 2011; Bhattacharyya et al., 2014). The 19S regulatory particle acts as a receptor, assisting in ubiquitination and unfolding of ubiquitinated protein substrates. Multiple catalytic sites degrade the substrate into short polypeptides, which are subsequently broken down by peptidases into peptides and amino acids that are recycled by cells (Tanaka et al., 1986; Hough et al., 1987).

The ubiquitin/26S proteasome pathway is widely involved in the regulation of plant development and growth (Silverstone et al., 2001; Tyler, 2004; Yu et al., 2015), signal transduction (Itoh et al., 2002; Potuschak et al., 2003; Smalle et al., 2003; Dill, 2004; Fleet and Sun., 2005), ATP-dependent degradation of ubiquitin (Clough et al., 1999; Hardtke et al., 2000; Schultz, 2001; Schwechheimer and Deng, 2001), and plant responses to biotic and abiotic stresses (Silverstone et al., 2001; Tyler, 2004; Fleet and Sun, 2005). A large body of evidence indicates that the $26 \mathrm{~S}$ proteasome is involved in the defense response of cells to pathogens. Some 26S proteasome subunits interact with pathogens effectors to inhibit their own activity and trigger PAMP-triggered immunity (PTI) and Effector-triggered Immunity (ETI) responses (Park et al., 2012; Üstün et al., 2014). For example, the effector protein AvrPiz-t of Magnaporthe grisea is degraded by the ubiquitin/proteasome pathway, which regulates PTI responses (Park et al., 2012). Moreover, effector proteins from the pathogen can target the proteasome. Üstün et al. (2014) reported that the 26S proteasome subunit RPT6 interacts with the effector protein HopZ4, which inhibits the activity of the $26 \mathrm{~S}$ proteasome making plants more susceptible to infection. In Arabidopsis, RPM1, a peripheral membrane protein mediates a hypersensitive response, and induction of RPM1 can increase plant defenses. Pathogen invasion shortens the half-life of RPM1, indicating that the ubiquitin/26S proteasome pathway is involved in defense against pathogens (Qiao et al., 2004). The yeast protein SGT1 was originally identified as a defense-related protein that could associate with SKP1 and CUL1, subunits of the Skp1-Cullin-F-box ubiquitin ligase complex (SCF) (Boyes et al., 1998). The orthologous plant protein SGT1 is also involved in the early defense response against pathogens, and entry of a pathogen induces attachment of the SGT1-SCF complex to the target protein, mediating its connection and degradation in Arabidopsis (Azevedo et al., 2002).

Programmed cell death plays a critical role in plant disease resistance and is triggered by resistance $(\mathrm{R})$ proteins (Coll et al., 2011). Vacuoles and plasma membranes mediated by the PBA1 subunit of the $26 \mathrm{~S}$ proteasome fuse together to release antimicrobial substances (Hatsugai et al., 2015). The Rho GTPase-activating protein SPIN6 interacted with U-box E3 ligase SPIN11 is also a key factor in the rice defense response (Liu et al., 2015). SPL11 promotes the ubiquitination of SPIN6, and SPIN6-RNAi plants show enhanced resistance to Xanthomonas oryzae and Magnaporthe oryzae (Liu et al., 2015). Arabidopsis botrytis susceptible1 (BO1) interacts with the MYB transcription factor BOS1, involved in the Arabidopsis defense response (Luo et al., 2010). BO1 can also ubiquitinate BOS1, and both BOS1 and its homologous genes can resist to the saprophytic fungus Botrytis cinerea in Arabidopsis (Luo et al., 2010). Since the $26 \mathrm{~S}$ proteasome system plays a crucial regulatory role in plant defense responses, it is a target for utilization by pathogens (Marino et al., 2012). The 26S proteasome 19S regulatory subunit RPN6 interacts with XopJ, a type III effector protein of Xanthomonas. RPN6 is induced by XopJ and transferred to the cell membrane. XopJ has protease activity to specifically degrade RPT6, leading to reduced proteasome activity. Inhibition of $26 \mathrm{~S}$ proteasome leads to abnormal vesicle trafficking, callus reduction, and salicylic acid (SA) signal transduction (Üstün et al., 2013; Üstün and Börnke, 2015).

Reactive oxygen species (ROS) play a key role in plant disease resistance. It is obvious that chemical substances such as ammonium diphenyliodonium chloride inhibit the accumulation of ROS during attacking by microbial pathogens, and ammonium diphenyliodochloride is thought to inhibit the accumulation of ROS-producing NADPH oxidase (Jabs et al., 1997). Moreover, ROS-producing systems triggered plant defense mechanism in plant (Wu et al., 1997). In our previous study, the bHLH transcription factor GmPIB1 
(P. sojae-inducible bHLH transcription factor) reduces the accumulation of ROS in cells by inhibiting expression of the peroxidase-related gene $G m S P O D$, thus improving the resistance of soybean hairy roots to P. sojae (Cheng et al., 2018). Here, we identified proteins interacting with GmPIB1 and tested whether they participate in the defense response to $P$. sojae, finding that the $26 \mathrm{~S}$ proteasome regulatory subunit GmPSMD interacts with GmPIB1. Overexpressing GmPSMD improved the activity of antioxidant enzymes simultaneously decreased ROS accumulation and enhanced resistance to $P$. sojae; conversely, knocking down GmPSMD increased susceptibility, and ROS accumulation and antioxidant enzymes activity was inhibited. These results indicated that GmPSMD improves the activity of antioxidant enzymes and suppresses ROS accumulation, suggesting that GmPSMD plays a positive regulatory role in the response of soybean to $P$. sojae.

\section{MATERIALS AND METHODS}

\section{Plant Materials and Growth Conditions}

The $P$ sojae-susceptible soybean cultivar "Dongnong 50 " and the resistant cultivar "Suinong10" were used. Seeds of the resistant cultivar "Suinong 10" were grown in an incubator at $25^{\circ} \mathrm{C}$, $70 \%$ relative humidity under a $16 \mathrm{~h}$ light $/ 8 \mathrm{~h}$ dark cycle, and the seedlings at stage V1 (the first true leaves were about to unfold; Fehr et al., 1971) were inoculated with P. sojae zoospores according to the methods described by Ward et al. (1979) and Yang et al. (1996) with minor modifications. Seeds of the susceptible cultivar "Dongnong 50" were obtained from the Key Laboratory of Soybean Biology in the Chinese Ministry of Education, Harbin, and this cultivar was used for genetic transformation experiments.

\section{Bioinformatics Analysis of GmPSMD}

The online database NCBI (National Center for Biotechnology Information) was used for finding the sequences of high homology genes with GmPSMD. DNAMAN software ${ }^{1}$ was used for the sequence multiple alignments, and a phylogenetic analysis of GmPSMD was carried out using MEGA software. The GmPSMD protein structure was predicted using Phyre $2^{2}$.

\section{RT-PCR and qRT-PCR Analysis}

Total RNA was isolated using Trizol reagent and reversetranscribed based on the manufacturer's instructions (Invitrogen, China). cDNA was synthesized from $1 \mu \mathrm{g}$ of total RNA using a Super Script first-strand cDNA synthesis system (Takara, Dalian, China). qRT-PCR was performed on a LightCycler96 instrument (Roche, Switzerland) using a real-time PCR kit (ToYoBo, Japan). The soybean GmEF1 $\beta$ housekeeping gene (GenBank accession no. NM_001248778) was used as an internal control to normalize all data (Supplementary Table S1). The relative transcript level of target genes was calculated using the

${ }^{1}$ http://www.lynnon.com/

${ }^{2}$ http://www.sbg.bio.ic.ac.uk/phyre2
$2^{-\Delta \Delta C T}$ method. Three biological repeats for each line were performed in each experiment.

\section{Yeast Two-Hybrid Assays}

Yeast two-hybrid assays were performed using the FrozenEZ Yeast Transformation II kit (The Epigenetics Company, United States). The full-length GmPSMD was inserted into the pGADT7 expression vector, and GmPIB1 was inserted into the pGBKT7 expression vector. Fusion plasmids pGADT7GmPSMD and pGBKT7-GmPIB1 were transformed into yeast strain $\mathrm{Y}_{2} \mathrm{HGold}$ (Takara Bio, Japan) to identify protein-protein interactions. pGBKT7-53 and pGADT7-SV40 plasmids were used as a positive control, while pGBKT7-Lam and pGADT7SV40 plasmids were used as the negative control in yeast cells.

\section{Luciferase Complementation Assays}

To construct GmPIB1-ccluc and GmPSMD-nluc, the coding sequences of GmPIB1 and GmPSMD were cloned into the plant expression vectors pCAMBIA1300-ccluc and pCAMBIA1300nluc using gene-specific primers, respectively (Supplementary Table S1). The recombinant plasmids were transferred into Agrobacterium tumefaciens GV3101, and transformed Agrobacterium strains resistant to kanamycin and rifampicin were selected and cultured in YEP medium (containing 0.5\% Yeast, $1 \%$ Peptone, $0.5 \% \mathrm{NaCl}$ ) at $28^{\circ} \mathrm{C}$ until the $\mathrm{OD}_{600}$ reached $0.8-1.2$. Agrobacterium cells were collected by centrifugation at 4,000 rpm for $10 \mathrm{~min}$, resuspended in infection liquid (containing $10 \mathrm{mM} \mathrm{MgCl}$, $10 \mathrm{~mm}$ MES, $150 \mu \mathrm{mol}$ acetosyringone, $\mathrm{pH}$ 5.6) and placed at room temperature $\left(25^{\circ} \mathrm{C}\right)$ for $2-3 \mathrm{~h}$. The two recombinant plasmids were mixed in equal volumes and injected into Nicotiana benthamiana. Fluorescence signals were observed after dark culture for 2 days.

\section{Induction and Purification of Fusion Proteins}

The open reading frames of GmPSMD and GmPIB1 were, respectively, fused to the GST-tag of vector PGEX-4T-1 and the $6 \times$ His-tag of vector pET29b $(+)$ (Novagen, Germany) and then transformed into Transetta (DE3) Escherichia coli cells (TransGen Biotech, China). GST-tagged and His-tagged proteins were induced with $0.5 \mathrm{mM}$ isopropyl- $\beta$-D-thiogalactoside (IPTG) at $37^{\circ} \mathrm{C}$ for $4 \mathrm{~h}$. GmPIB1-His and GmPSMDGST fusion proteins were purified using a GST-Sefinose kit (Sangon, China) or a His-bind Purification Kit (Merck Millipore) at $4^{\circ} \mathrm{C}$ and subsequently detected by sodium dodecyl sulfate polyacrylamide gel electrophoresis (SDS-PAGE) and immunoblotting using anti-His and anti-GST antibodies (Abmart, United States), respectively.

\section{GST Pull-Down Assays}

The GST fusion protein was fused with $50 \mu \mathrm{L}$ GST beads (Sangon, China) at $4^{\circ} \mathrm{C}$ for $1 \mathrm{~h}$ then washed three times with $1 \times$ phosphate-buffered saline (PBS) containing 1\% Triton100 before adding the purified fusion protein with His-tag and incubating at $4^{\circ} \mathrm{C}$ for $4 \mathrm{~h}$. The washing step was repeated, and 40 $\mu \mathrm{L} 5 \times$ loading buffer was added for immunoblotting detection. 


\section{Agrobacterium rhizogenes-Mediated Transformation of Soybean Hairy Roots}

To construct the pCAMBIA3301-GmPIB1 and pCAMBIA3301GmPSMD overexpression vector, the coding sequence of GmPIB1 and GmPSMD was cloned into the plant expression vector pCAMBIA3301 with a C-terminal $4 \times$ Myc fusion sequence, respectively. For RNA interference performance, the specific GmPSMD cDNA fragment was amplified and inserted into the vector PFGC5941 (Kerschen et al., 2004). "Dongnong 50" was used to generate transgenic soybean hairy roots by $A$. rhizogenes-mediated transformation following the instructions described by Paz et al. (2004). Transgenic soybean hairy roots were preliminarily detected by PCR (Primers shown in Supplementary Table S1), and then the level of gene overexpression or silencing were detected by qRT-PCR and those overexpressing GmPIB1 and GmPSMD were identified by immunoblotting with an anti-Myc antibody (Abmart, United States).

\section{Pathogen Response Assays of Transgenic Soybean Hairy Roots}

Resistance to $P$. sojae was assessed based on methods described by Ward et al. (1979) with minor modifications. GmPSMDtransformed "Dongnong 50" soybean hairy roots were cultured for about 2 weeks and were placed on a tray with clean and moist gauze. All hairy roots were slightly scratched at the same position with a sterile scalpel, and zoospores of $P$. sojae race 1 were used to inoculate the wounds. Empty vector (EV) soybean hairy roots were used as controls. Disease symptoms were recorded by photography. The P. sojae biomass was analyzed based on the accumulation of $P$. sojae TEF1 (GenBank accession no. EU079791), PSEL1 (GenBank accession no. CF840149), and PSEL2 (GenBank accession no. CF839332) in the transgenic soybean hairy roots. The pathogen response assays were performed on three biological replicates, each with three technical replicates.

\section{Detection of Total ROS and Antioxidant Enzyme Activity}

Total ROS production was determined according to the instructions of Reactive Oxygen Species Assay Kit (Beyotime Institute of Biotechnology, China). Fluorescence was detected at $530 \mathrm{~nm}$ emission wavelength and $485 \mathrm{~nm}$ excitation wavelength using a Microplate Reader (Bio-TEK, United States; Qian et al., 2009). For the enzyme assays, $0.2 \mathrm{~g}$ of soybean transgenic hairy roots was ground with $25 \mathrm{mM}$ HEPES buffer ( $\mathrm{pH}$ 7.8) containing $0.2 \mathrm{mM}$ EDTA, $2 \mathrm{mM}$ ascorbate and $2 \%$ PVP. The homogenate was centrifuged at $4^{\circ} \mathrm{C}$ for $15 \mathrm{~min}$ at $12,000 \times \mathrm{g}$ and the resulting supernatant was used for the determination of the enzymatic activity following the method described by Cao et al. (2009). The superoxide dismutase (SOD) activity was measured as the increase in the absorbance at $560 \mathrm{~nm}$ according to instructions of Superoxide Dismutase Assay Kit (COMIN, Institute of Biotechnology, Suzhou of China). The catalase (CAT) activity was measured as the decline in the absorbance at $240 \mathrm{~nm}$ due to the decrease of extinction according to the instructions of Superoxide Dismutase Assay Kit (COMIN, Institute of Biotechnology, Suzhou of China). The guaiacol peroxidase (GPX) activity was measured as the increase in the absorbance at $340 \mathrm{~nm}$ due to guaiacol oxidation according to the instructions of Superoxide Dismutase Assay Kit (COMIN, Institute of Biotechnology, Suzhou of China). The peroxidase (POD) activity was measured as the increase in the absorbance at $470 \mathrm{~nm}$ according to the instructions of Superoxide Dismutase Assay Kit (COMIN, Institute of Biotechnology, Suzhou of China). The detection of total ROS and antioxidant enzyme activity assays were performed on three biological replicates, each with three technical replicates.

\section{Detection of $\mathrm{H}_{2} \mathrm{O}_{2}$ and $\mathrm{O}_{2}{ }^{-}$}

Hydrogen peroxide $\left(\mathrm{H}_{2} \mathrm{O}_{2}\right)$ accumulation was determined according to the method of Velikova et al. (2000). Superoxide anion $\left(\mathrm{O}_{2}{ }^{-}\right)$accumulation in GmPSMD-transformed and EV soybean hairy roots was determined as follows: soybean hairy roots were inoculated with $P$. sojae to induce production of $\mathrm{O}_{2}{ }^{-}$, and samples were taken at 0,12 , and $24 \mathrm{~h}$. Then the samples were thoroughly ground and vortexed, and $500 \mu \mathrm{L}$ of $10 \mathrm{mM}$ phosphate buffer $(\mathrm{PH}=7.8)$ was added. After centrifuging at $4^{\circ} \mathrm{C}$ and 5,000 rpm for $10 \mathrm{~min}$, the supernatant was added with $700 \mu \mathrm{L}$ phosphate buffer and $100 \mu \mathrm{L}$ of $10 \mathrm{mM}$ hydroxylamine hydrochloride at $25^{\circ} \mathrm{C}$ for $20 \mathrm{~min}$. Finally, $0.5 \mathrm{~mL}$ of P-aminobenzenesulfonic acid and $0.5 \mathrm{~mL}$ of naphthylamine were added and reacted at $25^{\circ} \mathrm{C}$ for $20 \mathrm{~min}$ to read the absorbance of the supernatant at $530 \mathrm{~nm}$.

\section{Determination of 26S Proteasome Activity}

The $26 \mathrm{~S}$ proteasome activity assays were performed as previously described by Kisselev and Goldberg (2005). Briefly, GmPSMD and GmPIB1 transgenic soybean hairy roots were inoculated with $P$. sojae and the samples were taken at 0,12 , and $24 \mathrm{~h}$. The proteasome activity buffer $50 \mathrm{Mm}$ Tris- $\mathrm{HCl}(\mathrm{pH}=7.5)$, $250 \mathrm{mM}$ sucrose, $5 \mathrm{mM} \mathrm{MgCl} 2,0.5 \mathrm{mM}$ EDTA, $2 \mathrm{mM}$ ATP, $1 \mathrm{mM}$ DTT, $1 \%$ of the total volume of PSMF and Roche inhibitor were prepared and added to the sample in proportion. The homogenate was centrifuged at $4^{\circ} \mathrm{C}$ for $15 \mathrm{~min}$ at $10,000 \times \mathrm{g}$ and the resulting supernatant was used for the determination of $26 \mathrm{~S}$ proteasome activity. The supernatant, buffer and fluorescent substrate (proteasome substrate $\varnothing$, fluorogenic) were added to a 96-well plate (BD Flacon) at a ratio of 10:137.5:2.5. Fluorescence was detected at $460 \mathrm{~nm}$ emission wavelength and $380 \mathrm{~nm}$ excitation wave length at $25^{\circ} \mathrm{C}$. The changes of fluorescence units in $10 \mathrm{~min}$ were recorded.

\section{RESULTS}

\section{Screening and Identification of GmPIB1-Interacting Proteins}

To explore the proteins interacting with GmPIB1, the transgenic soybean hairy roots overexpressing GmPIB1 were obtained using Agrobacterium rhizogenes-mediated 
transformation. Proteins interacting with GmPIB1 were screened by immunoprecipitation-mass spectrometry and verified by yeast two-hybrid library screening. pCAMBIA3301GmPIB1-myc recombinant vector was successfully constructed (Supplementary Figure S1A) and the immunoblotting analysis confirmed successful production of GmPIB1 in transgenic soybean hairy roots (Supplementary Figure S1B). Then the immunoprecipitation was conducted and the specific fluorescent signal was observed in positive hairy roots (Figure 1A), indicating that protein activity of GmPIB1 was good. Specific stripes for co-immunoprecipitating proteins were observed in the experimental group, showing that the precipitation complex contained not only the target protein GmPIB1, but also proteins that may interact with GmPIB1. Several stripes observed in the experimental group and not in the control by silver staining (Figure 1B) were removed for mass spectrometry. completed by Wuhan GenecreateBiological Engineering Company. A total of 392 proteins that might interact with GmPIB1 were retrievaled and analyzed by Proteinpilot software, which were mainly related to energy metabolism, gene expression regulation, and transportion. Among the 392 proteins, 20 candidate genes were selected based on the identification score of mass spectrometry data (Supplementary Table S2). To verify interactions between proteins encoded by the candidate genes and GmPIB1, the full-length GmPIB1 gene was inserted into vector pGBKT7 and the candidate genes into vector pGADT7. Recombinant vectors were transformed into yeast strain $\mathrm{Y}_{2} \mathrm{H}$ Gold and titrated on $\mathrm{SD} /$-Leu/-Trp and SD/-Ade/-His/-Leu/-Trp plates to verify protein-protein interactions. Only yeast co-transformed with pGADT7-284 (GmPSMD) and pGBKT7-GmPIB1 grew on $\mathrm{SD} /$-Ade/-His/-Leu/-Trp plates (Supplementary Figure S2). Moreover, GmPSMD had no self-activating activity and the yeast two-hybrid results show that GmPSMD and GmPIB1 interact in yeast (Figure 1C).

\section{GmPIB1 and GmPSMD Interact in vitro and in vivo}

We further verified the interaction between GmPIB1 and GmPSMD using pull-down assays in vitro and luciferase complementation assays in vivo. The expression levels of the fusion proteins GmPIB1-HIS and GmPSMD-GST were very high at 1,2 , and $4 \mathrm{~h}$ after IPTG induction (Figure 2A), indicating that the proteins were well purified and could be used for pulldown assays (Figure 2B). As shown by immunoblotting analysis, GmPSMD could interact with full-length GmPIB1 in vitro (Figure 2C). We then infiltrated Agrobacterium containing the recombinant plasmids pCAMBIA1300-GmPIB1-ccluc and pCAMBIA1300-GmPSMD-nluc into Nicotiana benthamiana leaves and observed uimioluminiscence at the infiltration areas
A

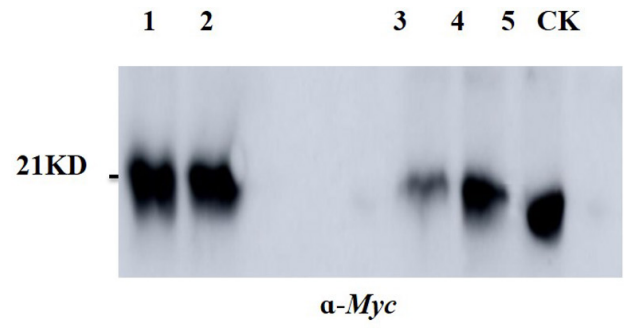

C
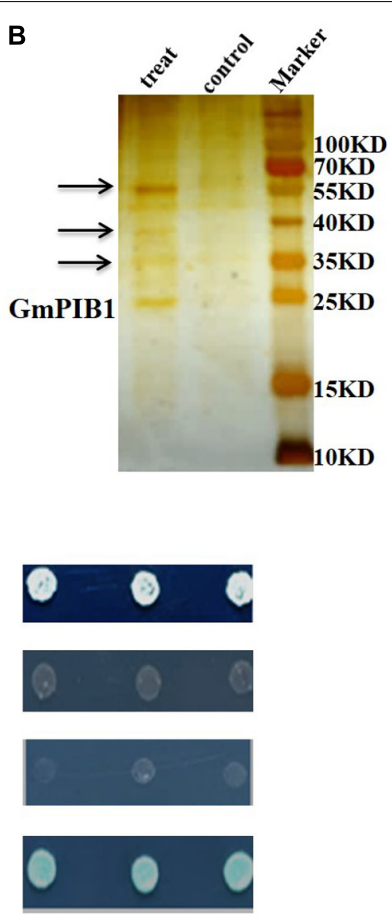

FIGURE 1 | Screening and identification of GmPIB1-interacting proteins. (A) Immunoprecipitation experiment. Lanes 1 and 2, protein supernatant from positive hairy roots; lane 3, immunoprecipitated protein supernatant from the experimental group; lane 4, immunoprecipitated protein supernatant from the control group; lane 5, experimental group immunoprecipitation products; CK, control group immunoprecipitation products. (B) Silver nitrate staining. Treat: immunoprecipitated products containing GmPIB1-Myc protein with anti-Myc; control: immunoprecipitated products containing GmPIB1-Myc protein with negative antibody (anti-Mouse); arrows indicate different bands. (C) Interaction of GmPIB1 with GmPSMD in yeast cells. Yeast strain $Y_{2}$ HGold transformed with fusion plasmids pGADT7-GmPSMD and pGBKT7-GmPIB1 was grown on SD/-Trp/-Leu media and SD/-Trp/-Leu/-His/-Ade media with added X- $\alpha$-gal. pGBKT7-53 and pGADT7-SV40 plasmids were used as a positive control; pGBKT7-Lam and pGADT7-SV40 plasmids were used as a negative control. 

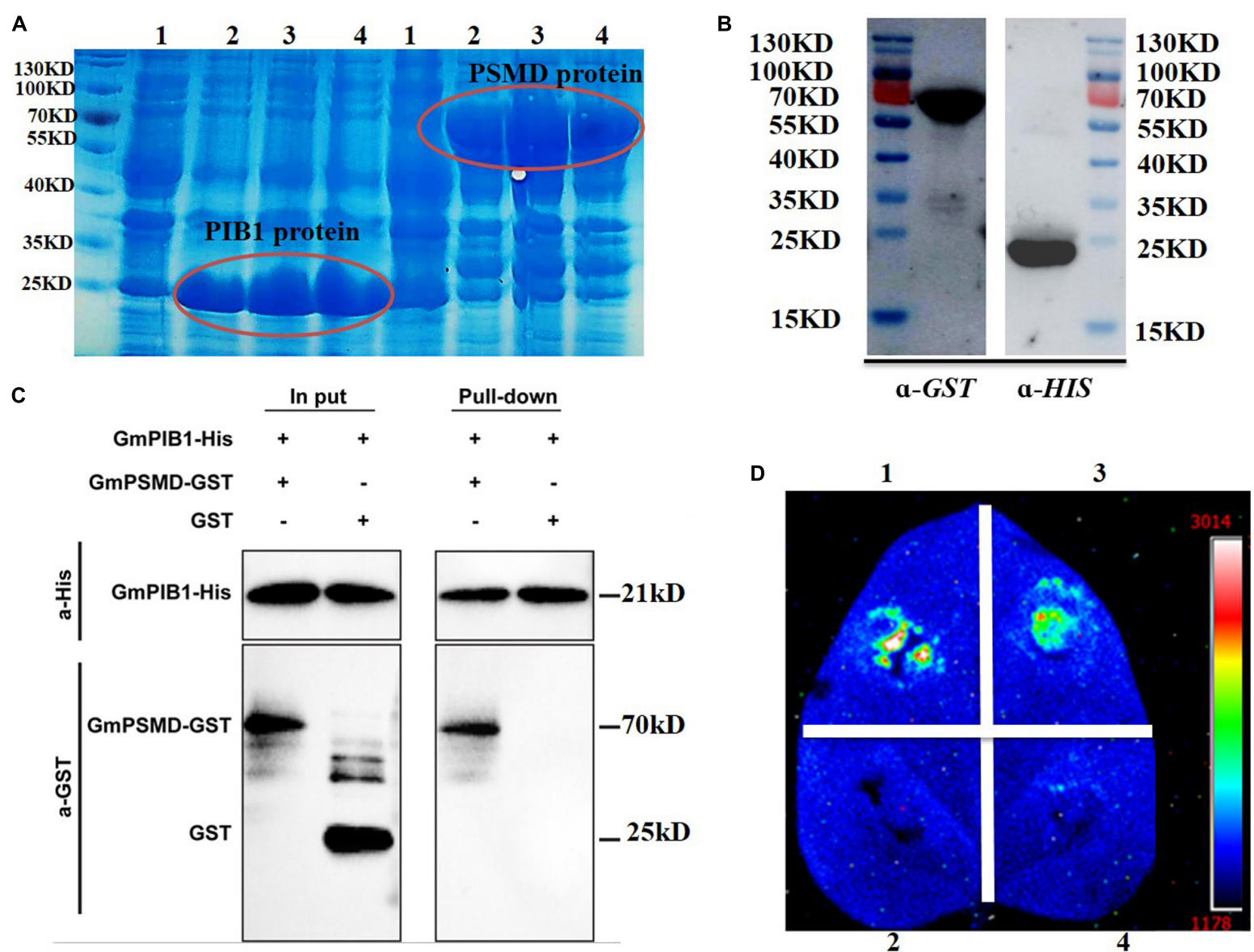

FIGURE 2 | Verification of interaction between GmPIB1 and GmPSMD. (A) Transetta cells containing pET29b-GmPIB1 and pGEX-4T-1-GmPSMD recombinant plasmids were grown at $37^{\circ} \mathrm{C}$ for 1,2 , or $4 \mathrm{~h}$ with or without $0.5 \mathrm{mM}$ isopropyl- $\beta$-D-thiogalactoside (IPTG) induction. Lane 1, GmPIB1-HIS and GmPSMD-GST proteins without IPTG induction; lane 2, GmPIB1-HIS and GmPSMD-GST proteins with IPTG induction for $1 \mathrm{~h}$; lane 3, GmPIB1-HIS and GmPSMD-GST proteins with IPTG induction for $2 \mathrm{~h}$; lane 4, GmPIB1-HIS and GmPSMD-GST proteins with IPTG induction for $4 \mathrm{~h}$. (B) Immunoblotting of purified recombinant GmPIB1 with anti-His antibody and GmPSMD with anti-GST antibody. (C) Pull-down verification of GmPIB1 interaction with GmPSMD. (D) Verification of GmPIB1 interaction with GmPSMD by luciferase complementation. Transient expression of recombinant pCAMBIA1300-GmPIB1-cluc and pCAMBIA1300-GmPSMD-nluc plasmids after co-injection into Nicotiana benthamiana. 1, positive control GB-nLuc + FIS2-cluc; 2, negative control nluc; 3, GmPIB1-cLuc + GmPSMD-nluc; 4, negative control cluc.

after dark incubation for 2 days, and the results showed that GmPIB1 and GmPSMD can interact in vivo (Figure 2D).

\section{Bioinformatics Analysis of GmPSMD}

GmPSMD is located on chromosome 6 with a full length of $1,803 \mathrm{bp}$ and a coding sequence of $1,269 \mathrm{bp}$; the encoded 423 amino acids contain a PCI domain belonging to the $26 \mathrm{~S}$ proteasome 19S regulatory subunit (Figure 3A). Phylogenetic tree and alignment analyses revealed that PSMD is divided into four sub-families, A, B, C, and D, among which soybean GmPSMD and Mucuna pruriens MPPSMD, Lupinus angustifolius LaPSMD, Abrus precatorius APPSMD, Vigna radiata. VrPSMD, Phaseolus vulgaris PvPSMD, Vigna angularis VaPSMD, and Cicer arietinum CaPSMD belong to subfamily A.

The amino acids of PSMD proteins from species belonging to the A subfamily in the phylogenetic tree were selected and analyzed by multi-column alignment using DNAMAN
(Figure 3B). The sequence similarity of GMPSMD with MpPSMD, LaPSMD, ApPSMD, VrPSMD, VaPSMD, CaPSMD, and PvPSMD was 70.91, 94.55, 95.97, 95.97, 96.21, 94.55, and 95.02\%, respectively (Figure 3C). GmPSMD had the highest similarity of $96.21 \%$ with VaPSMD and the lowest similarity of $70.91 \%$ with MPPSMD. The tertiary structure of GmPSMD was predicted using the website http://swissmodel.expasy.org. The protein consisted mainly of random coil, $\alpha$-helix, $\beta$-sheet and extended long chains. There were 20 alpha helices and four beta folds (Figure 3D).

\section{GmPSMD Affects Soybean Resistance to P. sojae}

To investigate whether GmPSMD is involved in the stress response induced by $P$. sojae, qRT-PCR was used to examine the transcript levels of GmPSMD in soybean cultivar "Suinong 10 " infected with P. sojae. The relative expression of GmPSMD 


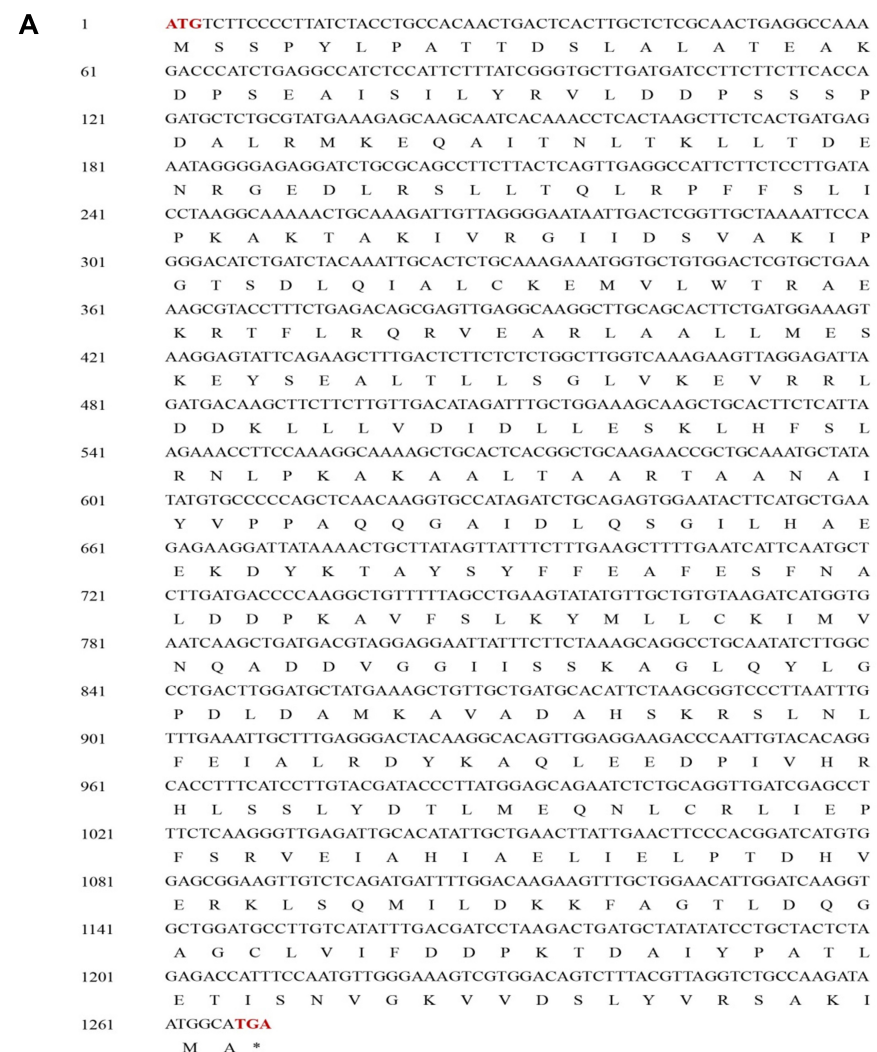

A

ATGTCTTCCCCTTATCTACCTGCCACAACTGACTCACTTGCTCTCGCAACTGAGGCCAAA $\begin{array}{lcccccccccccccccccccc} & \text { M } & \text { S } & \text { S } & \text { P } & \text { Y } & \text { L } & \text { P } & \text { A } & \text { T } & \text { T } & \text { D } & \text { S } & \text { L } & \text { A } & \text { L } & \text { A } & \text { T } & \text { E } & \text { A } & \text { K } \\ 61 & \text { GACCCATCTGAGGCCATCTCCATTCTTTATCGGGTCCTTGATGATCCTTCTTCTTCACCA }\end{array}$

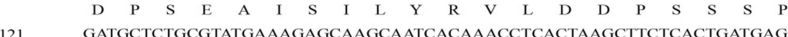
L R M K E Q A I T N L T K L L T D 181 AatAGGGGAGAGGATCTGCGCAGCCTTCTTACTCAGTTGAGGCCATTCTTCTCCTTGATA

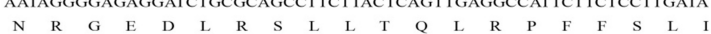
CCTAAGGCAAAAACTGCAAAGATTGTTAGGGGATAATTGACTCGGTTGCTAAAATTCCA CCTAAGGCAAAAACTGCAAAGATTGTTAGGGGAATAATTGACTCGGTTGCTAAAATTCCA $\begin{array}{llllllllllllllllllll}P & \text { K } & \text { A } & \text { K } & \text { T } & \text { A } & \text { K } & \text { I } & \text { V } & \text { R } & \text { G } & \text { I } & \text { I } & \text { D } & \text { S } & \text { V } & \text { A } & \text { K } & \text { I } & \text { P }\end{array}$

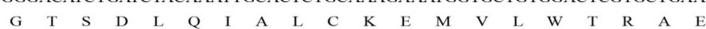
AAGCGTACCTTTCTGAGACAGCGAGTTGAGGCAAGGCTTGCAGCACTTCTGATGGAAAGT K $R$ T F L R Q  AAGGAGTATTCAGAAGCTHTGACTCTOCTCHCTGGOTHGGTCAAAGAAGTTAGGAGATAA GATGACAAGCTCHCTHGTHOACATAOATTGGTGGAAAGCAAGCTGCACTTCTCATTA $\begin{array}{lllllllllllllllllllll}\text { D } & \text { D } & \text { K } & \text { L } & \text { L } & \text { L } & \text { V } & \text { D } & \text { I } & \text { D } & \text { L } & \text { L } & \text { E } & \text { S } & \text { K } & \text { L } & \text { H } & \text { F } & \text { S } & \text { L }\end{array}$ AGAAACCTTCCAAAGGCAAAAGCTGCACTCACGGCTGCAAGAACCGCTGCAAATGCTATA

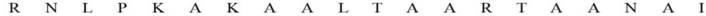
TATGTGCCCCCAGCTCAACAAGGTGCCATAGATCTGCAGAGTGGAATACTTCATGCTGAA $\mathrm{Y} V$ P P A Q Q G A I D L Q S G I L H A E

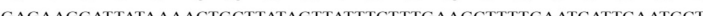

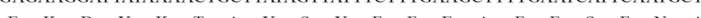
E K D $\mathrm{Y}$ K $\mathrm{T}$ A $\mathrm{Y}$ S $\mathrm{Y}$ P $\mathrm{F}$ E A $\mathrm{T}$ E $\mathrm{S}$ T $\mathrm{N}$ A CTTGATGACCCCAAGGCTGTHTTAGCCTGAAGTATATGTHGCTGTOTAAGATCATGGTC $\begin{array}{llllllllllllllllllll}\text { L } & \text { D } & \text { D } & \text { P } & \text { K } & \text { A } & \text { V } & \text { F } & \text { S } & \text { L } & \text { K } & \text { Y } & \text { M } & \text { L } & \text { L } & \text { C } & \text { K } & \text { I } & \text { M } & \text { V }\end{array}$ AATCAAGCTGATGACGTAGGAGGAATTATTTCTTCTAAAGCAGGCCTGCAATATCTTGGC $\begin{array}{lllllllllllllllllllll}\text { N } & \text { Q } & \text { A } & \text { D } & \text { D } & \text { V } & \text { G } & \text { G } & \text { I } & \text { I } & \text { S } & \text { S } & \text { K } & \text { A } & \text { G } & \text { L } & \text { Q } & \text { Y } & \text { L } & \text { G }\end{array}$ CCTGACTTGGATGCTATGAAAGCTGTTGCTGATGCACATTCTAAGCGGTCCCTTAATTTG

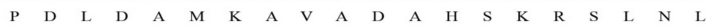
TTTGAAATTGCTTTGAGGGACTACAAGGCACAGTTGGAGGAAGACCCAATTGTACACAGG

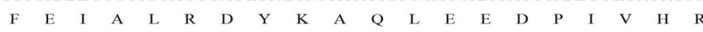
CACCTTTCATCCTTGTACGATACCCTTATGGAGCAGAATCTCTGCAGGTTGATCGAGCCT $\begin{array}{llllllllllllllllllll}\text { H } & \text { L } & \text { S } & \text { S } & \text { L } & \text { Y } & \text { D } & \text { T } & \text { L } & \text { M } & \text { E } & \text { Q } & \text { N } & \text { L } & \text { C } & \text { R } & \text { L } & \text { I } & \text { E } & \text { P }\end{array}$ TTCTCAAGGGTTGAGATTGCACATATTGCTGAACTTATTGAACTTCCCACGGATCATGTG

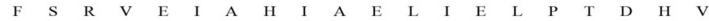
GAGCGGAAGTTGTCTCAGATGATTTTGGACAAGAAGTTTGCTGGAACATTGGATCAAGGT $\begin{array}{llllllllllllllllllllll}\text { E } & R & K & \text { L } & \text { S } & \text { Q } & \text { M } & \text { I } & \text { L } & \text { D } & \text { K } & \text { K } & \text { F } & \text { A } & \text { G } & \text { T } & \text { L } & \text { D } & \text { Q } & \text { G }\end{array}$ GCTGGATGCCTTGTCATATTTGACGATCCTAAGACTGATGCTATATATCCTGCTACTCTA $\begin{array}{llllllllllllllllllll}\text { A } & G & \text { C } & \text { L } & \text { V } & \text { I } & \text { F } & \text { D } & \text { D } & \text { P } & \text { K } & \text { T } & \text { D } & \text { A } & \text { I } & \text { Y } & \text { P } & \text { A } & \text { T } & \text { L }\end{array}$ GAGACCATTTCCAATGTTGGGAAAGTCGTGGACAGTCTTTACGTTAGGTCTGCCAAGATA $\begin{array}{lllllllllllllllllllll} & \text { E } & \text { T } & \text { I } & \text { S } & \text { N } & \text { V } & \text { G } & \text { K } & \text { V } & \text { V } & \text { D } & \text { S } & \text { L } & \text { Y } & \text { V } & \text { R } & \text { S } & \text { A } & \text { K } & \text { l }\end{array}$ ATGGCATGA

C Vigna radiata_va Vigna_angularis Phaseolus vulga Iucuna pruriens Lupinus angustif Gossypium arbore Glycine max XP Cicer-arietinum Abrus precatoriu Consensus
B

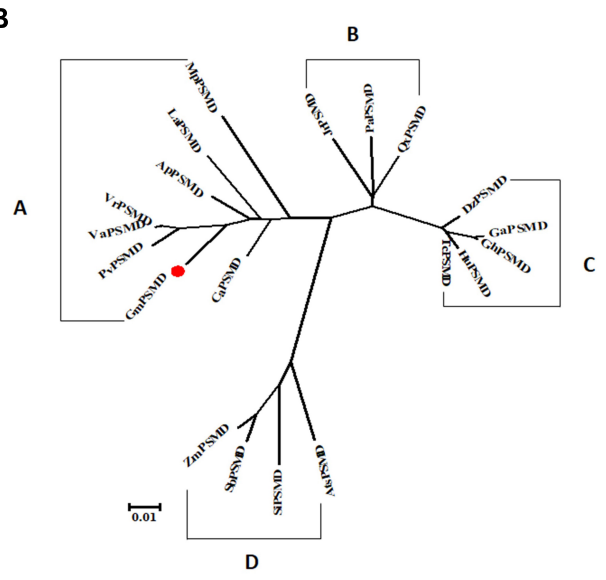

D

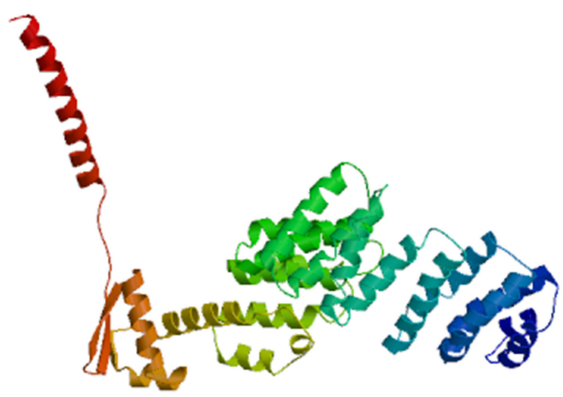

FIGURE 3 | Sequence and structure of GMPSMD. (A) Nucleotide and amino acid sequences of GmPSMD cDNA. (B) Phylogenic analysis of GmPSMD and other PSMD proteins. GenBank accession numbers are as follows: GmPSMD (XP_014631720), MpPSMD (RDY13590), LaPSMD (XP_019417640), ApPSMD (XP_027354762), VrPSMD (XP_014501695), VaPSMD (XP_017409210), CaPSMD (XP_012571699), PvPSMD (XP_007137150), JrPSMD (XP_018836824), PaPSMD (XP_021807467), QsPSMD (XP_023895672), SiPSMD (XM_004963066.3), SbPSMD (XP_002446538), AtPSMD (XM_020338016.1), ZmPSMD (XM_020547787.1), GaPSMD (XP_017637832), GhPSMD (AXQ39585), HuPSMD (XP_021299831), TMD (EOY01828). (C) Alignment of the GmPSMD amino acid sequence with other sequences in subfamily A of PSMD. (D) Predicted three-dimensional structure of GmPSMD.

increased after inoculation with $P$. sojae race 1 and reached the highest level after $36 \mathrm{~h}$ (Figure 4A). This indicated that GmPSMD may be involved in the defense response against $P$. sojae.

To further explore the defense response of GMPSMD against P. sojae. GmPSMD-OE and GmPSMD-RNAi transgenic soybean hairy roots were obtained and the level of overexpression and silencing of GmPSMD transgenic soybean hairy roots were analyzed by qRT-PCR. The relative expression of GmPSMD in GmPSMD-OE transgenic soybean hairy roots was remarkably higher than that in control, while it showed lower level than control in GmPSMD-RNAi transgenic soybean hairy roots (Figure 4B). Characterization of disease resistance in GmPSMD transgenic soybean roots of the susceptible cultivar "Dongnong 50 " revealed that the GmPSMD-OE soybean hairy roots turned slightly brown, while those transformed with pCAMBIA3301 empty vector and GmPSMD-RNAi showed rotting and browning of the inoculated parts (Figure 4C), and the lesion areas of GmPSMD-OE transgenic soybean hairy roots were significantly smaller than that in the control, while the GmPSMD-RNAi soybean hairy roots were significantly larger than that in control (Figure 4D).

Moreover, the relative biomass of $P$. sojae based on transcript levels of the P. sojae TEF1 (GenBank accession no. EU079791), PSEL1 (GenBank accession no. CF840149), and PSEL2 (GenBank accession no. CF839332) in infected soybean hairy roots after 2 days of incubation with zoospores of $P$. sojae was significantly $\left.{ }^{* *} P<0.01\right)$ lower in GmPSMD-OE lines than in EV hairy roots. Conversely, the biomass of $P$. sojae in GmPSMD-RNAi roots was higher than that in the control (Figures $\mathbf{5 A - C}$ ). These results indicated that overexpression of GmPSMD can 


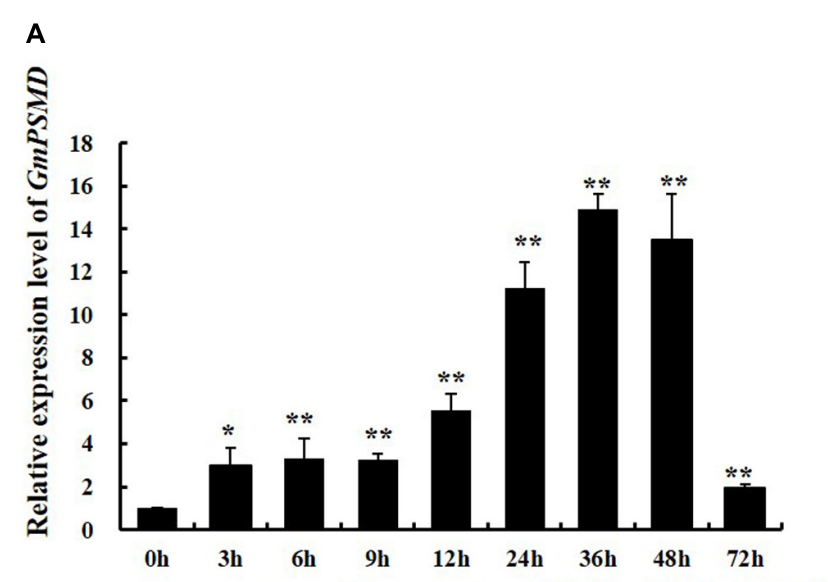

B

C
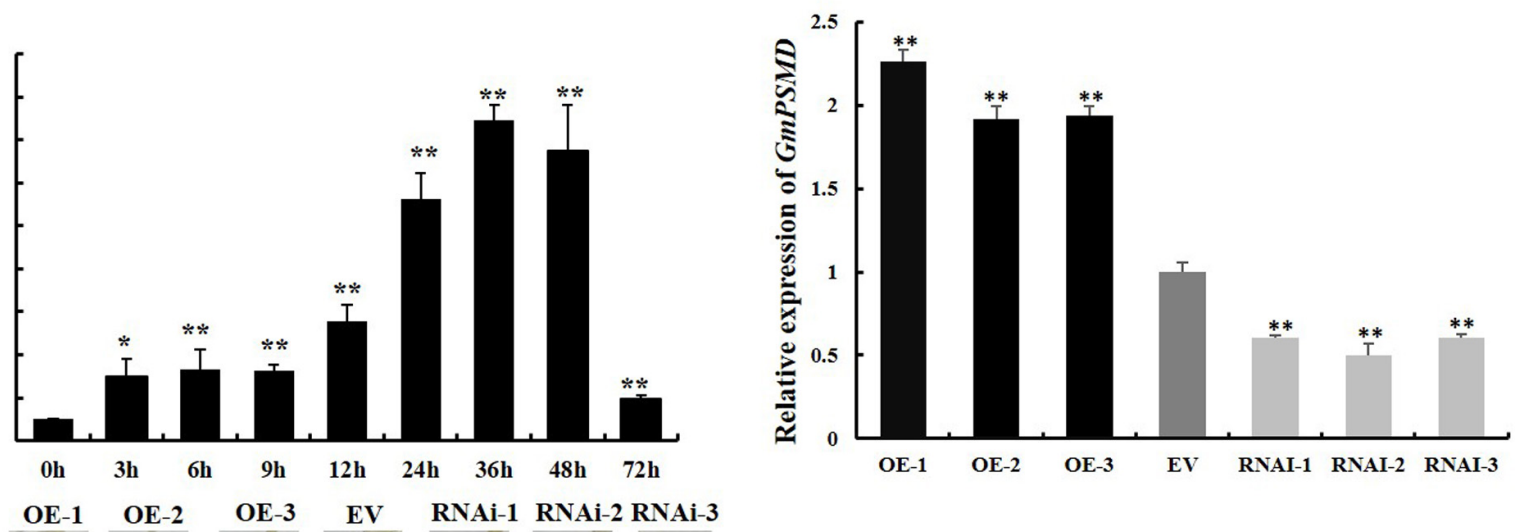

oh
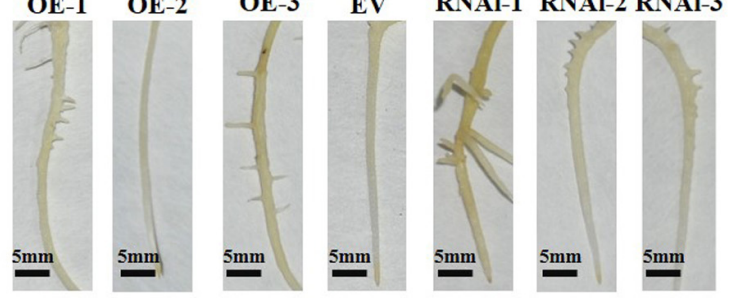

D

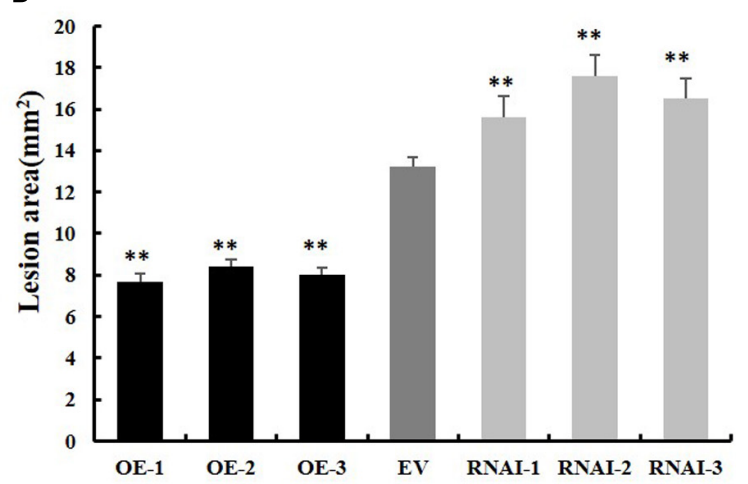

FIGURE 4 | Resistance to P. sojae in GmPSMD transgenic hairy roots. (A) Relative expression levels of GmPSMD in soybean cultivar "Suinong 10" upon P. sojae infection, determined by qRT-PCR. Plants of the resistant cultivar "Suinong 10" were grown until the first true leaf was about to unfold and were inoculated with $P$. sojae zoospores. The leaves were sampled at $0,3,6,9,12,24,36,48$, and $72 \mathrm{~h}$ after inoculation with $P$. sojae race 1. (B) qRT-PCR analysis of the relative expression of GmPSMD-OE, GmPSMD-RNAi transgenic hair roots and EV. Test strips for detection of transgenic soybean hairy roots. (C) Typical infection phenotypes of EV (control), GMPSMD-RNAi and GmPSMD-OE (overexpressing) soybean hairy roots after P. sojae inoculation. The soybean hairy roots were cultured for about 2 weeks and were inoculated with $P$. sojae zoospores. bars, $5 \mathrm{~mm}$. Hairy roots carrying empty vector (EV) were used as controls. (D) Lesion size measured from transgenic GmPSMD-OE, GmPSMD-RNAi transgenic hairy roots and EV at $72 \mathrm{~h}$ post-inoculation (hpi). The lesion size of each independent soybean line $(n=3)$ was calculated. Three biological replicates, each containing three technical replicates, were averaged and statistically analyzed by Student's $t$-test ${ }^{*} P<0.05$; $\left.{ }^{\star \star} P<0.01\right)$. Bars show standard error of the mean.

improve the resistance of soybean hairy roots to $P$. sojae, while GmPSMD-RNAi transgenic soybean hairy roots showed increased susceptibility to $P$. sojae.

\section{GmPSMD May Affect Soybean Resistance to $P$. sojae by Reducing the Level of ROS}

ROS are key signaling molecules in the interaction between plants and pathogens under stress (Mittler et al., 2004; Shigeoka and Maruta, 2014). To determine if GmPSMD affects the resistance of soybean hairy roots to $P$. sojae by affecting the production of ROS, the relative ROS levels in GmPSMD-RNAi, GmPSMD-OE, and EV transgenic soybean hairy roots were analyzed at 0,12 , and $24 \mathrm{~h}$ after inoculation with $P$. sojae. Accumulation of ROS in GMPSMD-RNAi hairy roots reached significant levels after $24 \mathrm{~h}$ compared with that in control, while ROS accumulation in GmPSMD-OE hairy roots was lower than that in control (Figure 6A).

ROS include hydrogen peroxide $\left(\mathrm{H}_{2} \mathrm{O}_{2}\right)$ and superoxide anions $\left(\mathrm{O}_{2}^{-}\right)$. Therefore, the contents of $\mathrm{H}_{2} \mathrm{O}_{2}$ and $\mathrm{O}_{2}{ }^{-}$ were further analyzed after inoculation with $P$. sojae in $\mathrm{EV}$, GmPSMD-OE, and GmPSMD-RNAi transgenic soybean hairy roots. The relative $\mathrm{H}_{2} \mathrm{O}_{2}$ levels in all three transgenic soybean hairy roots increased gradually with the extension of inoculation time. However, the accumulation of $\mathrm{H}_{2} \mathrm{O}_{2}$ in GmPSMD-OE transgenic soybean hairy roots was lower than that in the control, while $\mathrm{H}_{2} \mathrm{O}_{2}$ accumulation in transgenic soybean hairy roots of GmPSMD-RNAi was higher than that in the control 


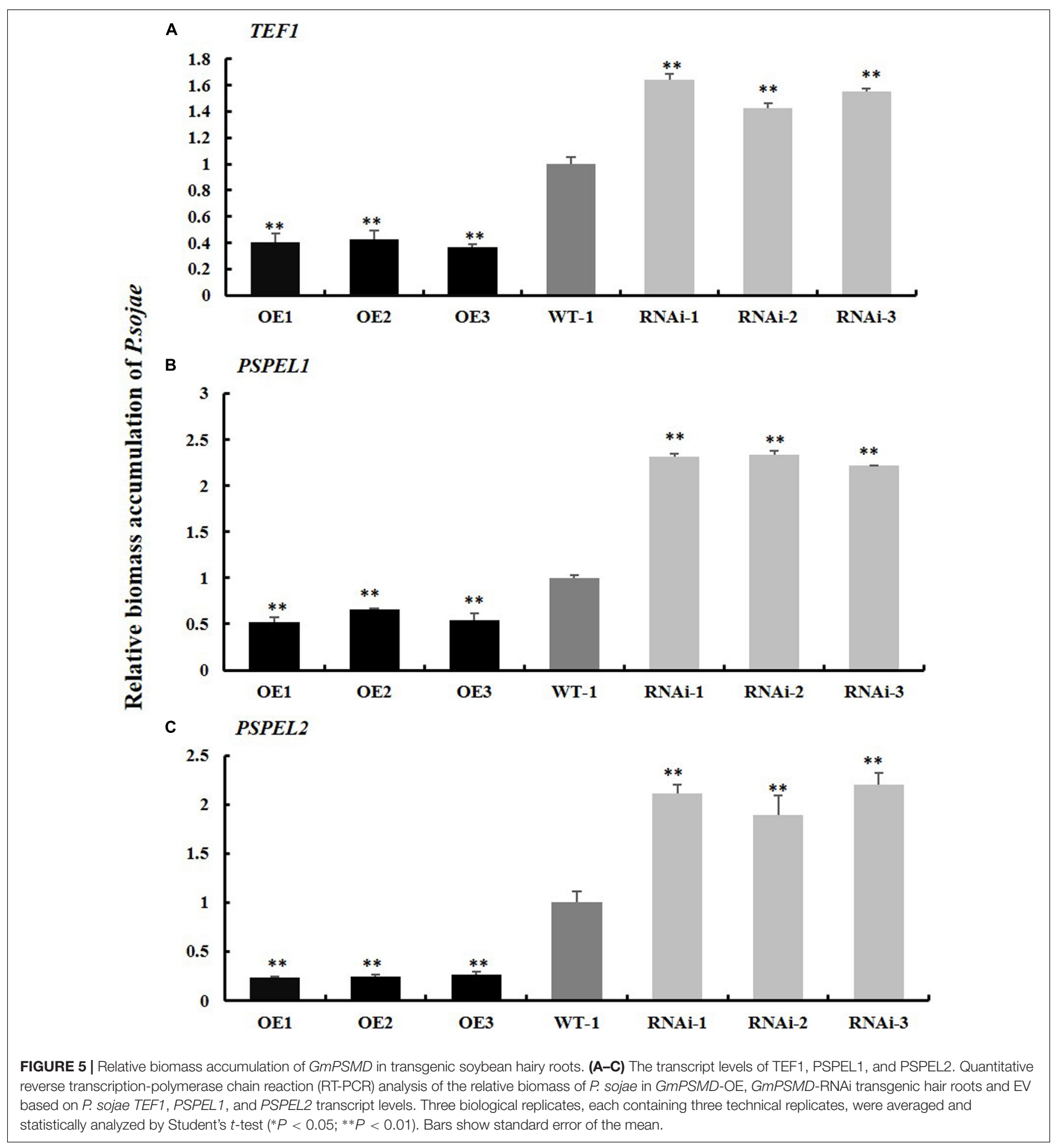

(Figure 6B). Levels of $\mathrm{O}_{2}{ }^{-}$in transgenic soybean hairy roots were not remarkably different from those of the control (Figure 6C). SOD, POD, CAT and GPX as the main antioxidant enzymes participated in the scavenging of reactive oxygen species and alleviated the damage of membrance system (Zhang et al., 2008). The antioxidant enzymes activity of SOD, POD, CAT, and GPX were analyzed after inoculation with P. sojae in EV,
GmPSMD-OE, and GmPSMD-RNAi transgenic soybean hairy roots. The antioxidant enzymes activity increased gradually with the extension of inoculation time. However, the antioxidant enzymes activity in GmPSMD-OE transgenic soybean hairy roots was higher than that in control, while the antioxidant enzymes activity in transgenic soybean hairy roots of GMPSMD-RNAi was lower than that in the control (Figure 7). 


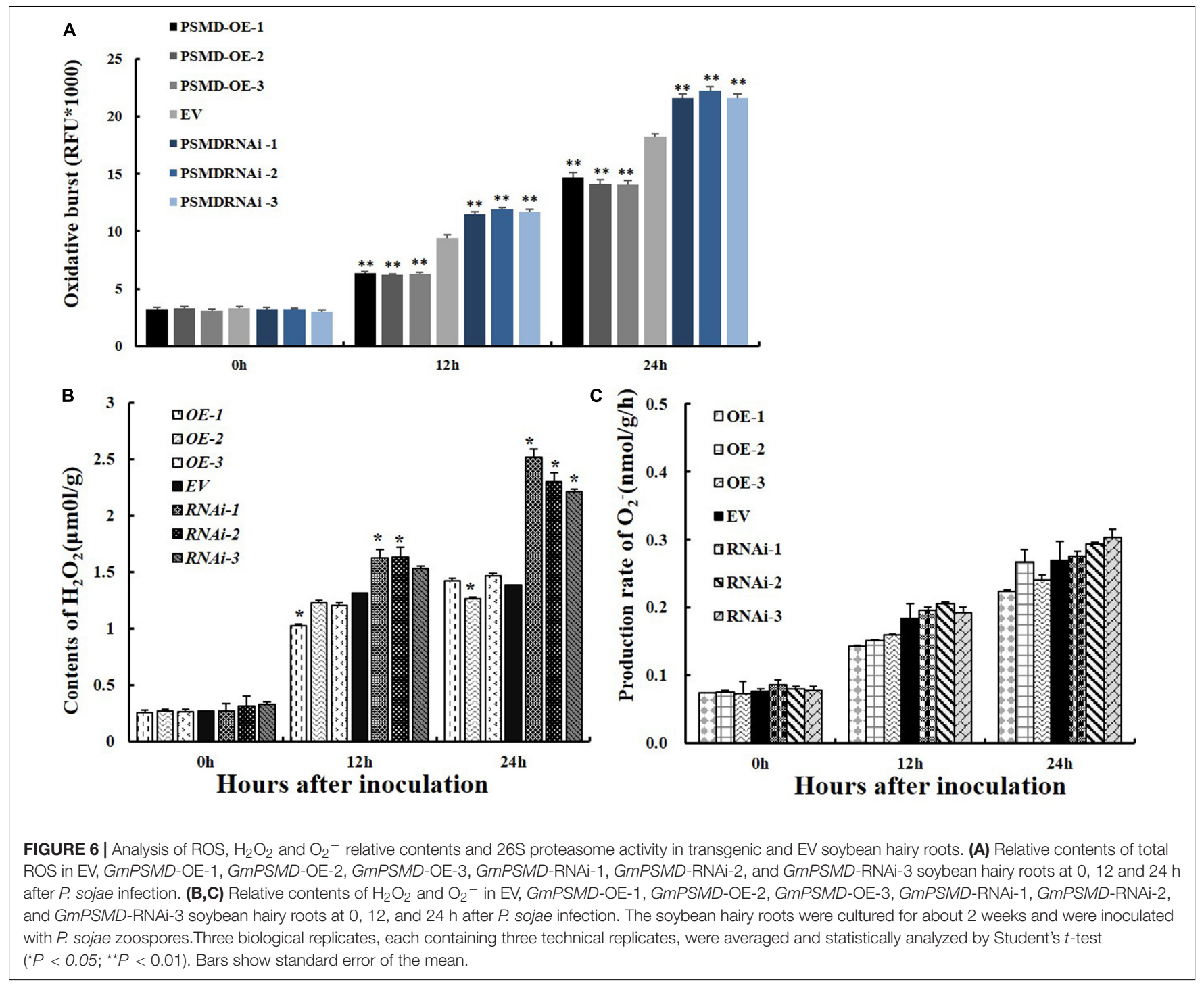

\section{GmPSMD May Affect 26S Proteasome Activity in GmPSMD and GmPIB1 Transgenic Soybean Hairy Roots During $P$ sojae Infection}

To verify if GmPSMD affects $26 \mathrm{~S}$ proteasome activity of transgenic soybean hairy roots and explore its role in response to $P$. sojae infection. The $26 \mathrm{~S}$ proteasome activity was measured in GmPSMD-OE, GmPSMD-RNAi, GmPIB1-OE, and EV transgenic soybean hairy roots inoculated with $P$. sojae. The results showed that the $26 \mathrm{~S}$ proteasome activity in hairy roots of GmPSMD-OE and GmPIB1-OE was remarkably higher than that in control, while the $26 \mathrm{~S}$ proteasome activity in GmPSMD-RNAi soybean hairy roots was remarkably lower than that in the control after infection with $P$. sojae (Figure 8A). These results indicate that GmPSMD could increase the $26 \mathrm{~S}$ proteasome activity in transgenic soybean hairy roots during $P$. sojae infection. We also found that the expression levels of GmPIB1 in GmPSMD-OE transgenic soybean hairy roots and GmPSMD in GmPIB1-OE transgenic soybean hairy roots increased, respectively, which proved that they were a pair of positive regulatory factors (Figures 8B,C).

\section{DISCUSSION}

Complex plant innate immune system networks have gradually developed with the evolution of plants and pathogens, and include the pathogen-associated molecular pattern-triggered immune response caused by pathogen-related molecular models and the effector-triggered immune response (Jones and Dangl, 2006). Identification of genes related to $P$. sojae infection has helped our understanding of the genetic mechanism of the response against Phytophthora root and stem rot of soybean (Xu et al., 2014; Cheng et al., 2015; Kong et al., 2015; Zhang et al., 2017). The bHLH transcription factor GmPIB1 is a positive regulator that responds to $P$. sojae infection (Cheng et al., 2018). 

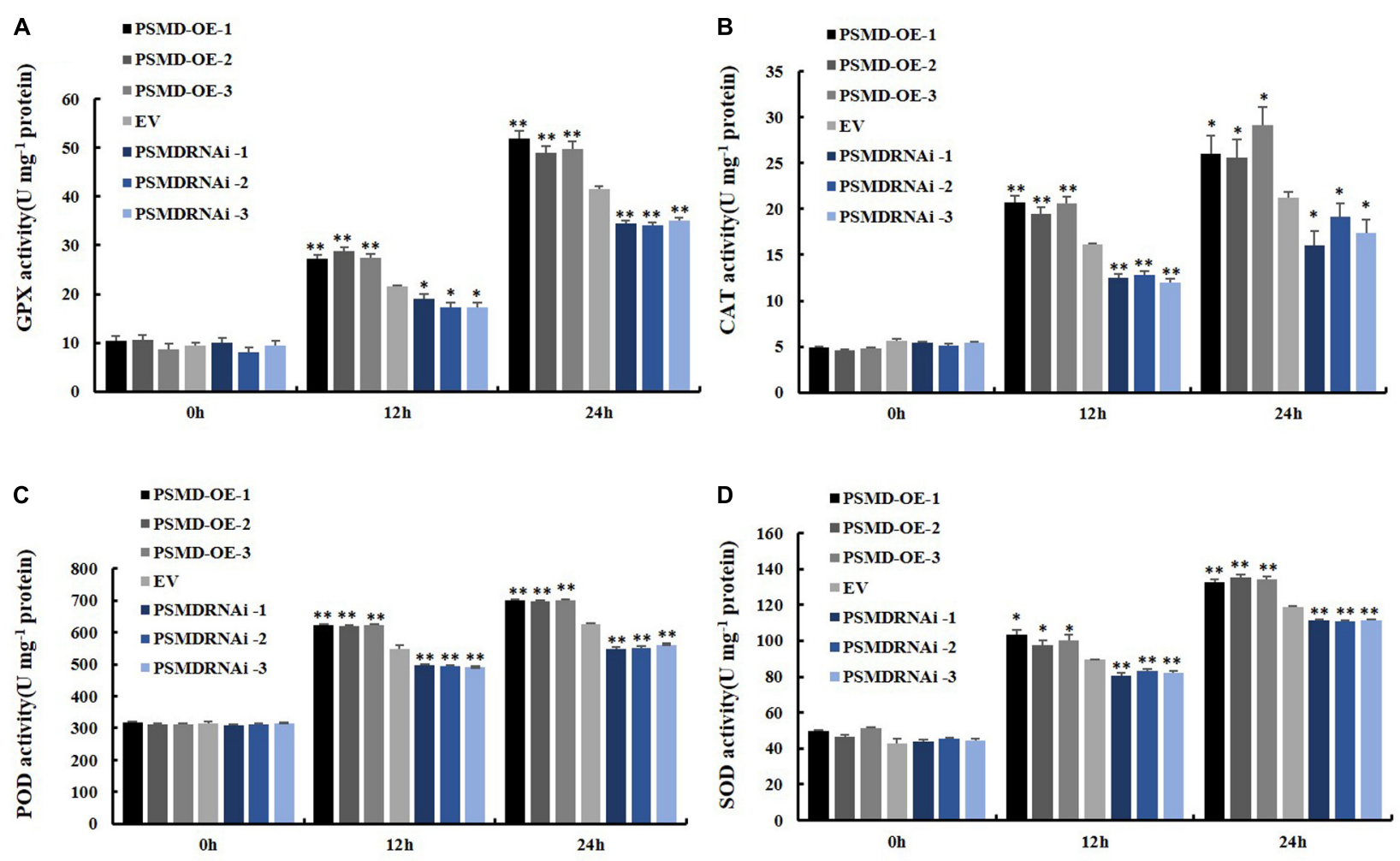

FIGURE 7 | Determination of antioxidant enzyme activity. (A) Determination of antioxidant enzyme activity of GPX. (B) Determination of antioxidant enzyme activity of CAT. (C) Determination of antioxidant enzyme activity of POD. (D) Determination of antioxidant enzyme activity of SOD. The soybean hairy roots were cultured for about 2 weeks and were inoculated with $P$. sojae zoospores. Three biological replicates, each containing three technical replicates, were averaged and statistically analyzed by Student's $t$-test $\left({ }^{\star} P<0.05\right.$; $\left.{ }^{* \star} P<0.01\right)$. Bars show standard error of the mean.

In this study, we identified the GmPSMD protein interacting with GmPIB1 using mass spectrometry. GmPSMD is a component of the $26 \mathrm{~S}$ proteasome regulatory subunits. Several studies have demonstrated that the $26 \mathrm{~S}$ proteasome is involved in the resistance of rice and Arabidopsis to pathogens (Qiao et al., 2004; Park et al., 2012; Hatsugai et al., 2015). The 26S proteasome subunits can interact with pathogens effectors such as HopZ4, AvrPiz-t to inhibit their own activity and trigger PTI and ETI responses (Park et al., 2012; Üstün et al., 2014). They can also degrade ubiquitinated modified proteins in the ubiquitin/26S proteasome pathway to participate in disease response (Luo et al., 2010; Liu et al., 2015). Although the 26S proteasome is widely involved in the resistance response of plants to pathogens, little is known about its involvement in the defense response to $P$. sojae. As GmPIB1 is a positive regulator of this response, it is likely that GmPSMD also participates and affects the resistance of soybean to P. sojae. Expression of GmPSMD in soybean was significantly increased after infection by $P$. sojae, indicating that GMPSMD might participate in the process of soybean resistance to $P$. sojae (Figure 4A). Furthermore, overexpression of GmPSMD significantly improved soybean resistance to $P$. sojae, while GmPSMD-RNAi produced the opposite symptoms (Figure 4C). The biomass accumulation of P. sojae in GmPSMD-RNAi transgenic soybean hairy roots was higher than that in controls byqRT-PCR (Figure 5), further proving that GmPSMD regulates soybean resistance to $P$. sojae. These findings suggest that GmPSMD plays a significant role in defense against $P$. sojae in soybean hairy roots.

ROS are important signaling molecules regulating plant responses to biological stress, including the process of plantpathogen interaction (Hückelhoven and Kogel, 2003). They are produced not only by primary metabolism, but also by apoplast-localized oxidases or peroxidases and plasma membrane (Suzuki et al., 2011; Cheng et al., 2015; Zhang et al., 2015, 2016). Evidence for the role of ROS during attack by microbial pathogens was provided by inhibition of ROS accumulation and plant defense by chemicals like diphenylene iodonium chloride which is thought to suppress a ROS-producing NADPH oxidase (Jabs et al., 1997). GmPIB1 is a positive regulatory factor in the response to $P$. sojae infestation, and ROS levels in GmPIB1-OE, GmPIB1-RNAi and EV transgenic soybean hairy roots indicated that GmPIB1 affects soybean resistance to $P$. sojae through regulating ROS levels (Cheng et al., 2018). We therefore proposed that GmPSMD might improve the resistance of transgenic soybean hairy roots to $P$. sojae by affecting levels of ROS and determined the levels of ROS in EV, GmPSMD-OE and GmPSMD-RNAi transgenic soybean hairy roots. The results indicated that GMPSMD suppresses ROS accumulation (Figure 6A), which is consistent with our expectation. 

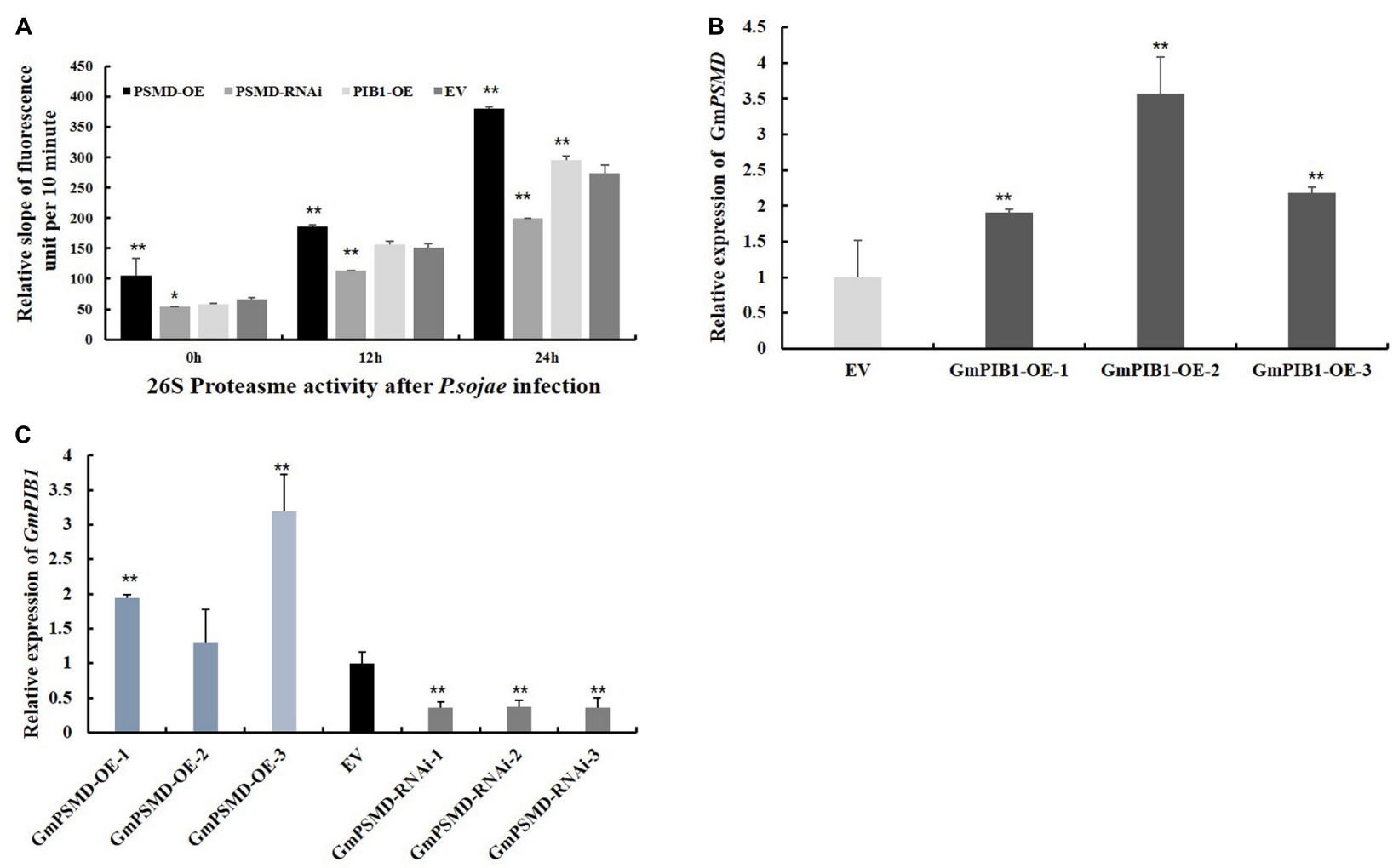

FIGURE 8 | 26S proteasome activity and the transcript levels of GmPIB1 and GMPSMD in transgenic and EV soybean hairy roots. (A) The 26S proteasome activity in GmPSMD-OE, GmPSMD-RNAi and GmPIB1-OE transgenic and EV soybean hairy roots inoculated with $P$. soaje was determined. The soybean hairy roots were cultured for about 2 weeks and were inoculated with $P$. sojae zoospores. The slope of fluorescence units per 10 min represents the activity of $26 S$ proteasome. The proteasome substrate $\varnothing$, fluorogenic was used as fluorescent substrate. Fluorescence was detected at $460 \mathrm{~nm}$ emission wavelength and $380 \mathrm{~nm}$ excitation wavelength at $25^{\circ} \mathrm{C}$. The changes of fluorescence units in $10 \mathrm{~min}$ were recorded. Three biological replicates, each containing three technical replicates, were averaged and statistically analyzed by Student's $t$-test $\left({ }^{\star} P<0.05\right.$; $\left.{ }^{\star \star} P<0.01\right)$. Bars show standard error of the mean. The values on the bar chart are the average of three biological repetitions. (B) qRT-PCR analysis of the relative expression of GmPSMD in GmPIB1-OE and EV soybean hairy roots based on EF1 $\beta$ transcript levels. (C) qRT-PCR analysis of the relative expression of GmPIB1 in GmPSMD-OE, GmPSMD-RNAi, and EV soybean hairy roots based on EF1 $\beta$ transcript levels. Three biological replicates, each containing three technical replicates, were averaged and statistically analyzed by Student's $t$-test $\left({ }^{\star} P<0.05 ;{ }^{* \star} P<0.01\right)$. Bars show standard error of the mean.

Hydrogen peroxide $\left(\mathrm{H}_{2} \mathrm{O}_{2}\right)$ and superoxide anion $\left(\mathrm{O}_{2}{ }^{-}\right)$are the main components of ROS. Under stress, accumulation of $\mathrm{H}_{2} \mathrm{O}_{2}$ in plant cells kills pathogens and induces immune reactions. Meanwhile, lower concentrations of $\mathrm{H}_{2} \mathrm{O}_{2}$ can also act as signaling molecules to induce a series of genes encoding defense-reactive protein, increasing resistance to pathogens (Foyer and Shigeoka, 2011). We further analyzed levels of $\mathrm{H}_{2} \mathrm{O}_{2}$ and $\mathrm{O}_{2}{ }^{-}$using qRTPCR. The results showed that GmPSMD could reduce $\mathrm{H}_{2} \mathrm{O}_{2}$ accumulation (Figure 6B). However, it's remarkable that there was no obvious difference in the level of $\mathrm{O}_{2}{ }^{-}$between transgenic soybean hairy roots and the control (Figure 6C).

Antioxidant enzymes in plants are able to remove part of the reactive oxygen species in time and maintain the oxygen balance (Noctor and Foyer, 1998; Mittler et al., 2004). SOD, POD, CAT, and GPX as the main antioxidant enzymes participated in the scavenging of reactive oxygen species and alleviated the damage of membrance system (Zhang et al., 2008). The enzymes activity of SOD, POD, CAT, and GPX were further analyzed, and the results showed that GmPSMD could increase enzymes activity of SOD, POD, CAT, and GPX (Figure 7). Moreover, the activities of $26 \mathrm{~S}$ proteasome increased significantly in GmPSMD-OE and GmPIB1-OE transgenic soybean hairy roots comparing with that in control after infection with $P$. sojae (Figure 8A), which means that the activities of 26S proteasome might be critical for GmPSMD and GmPIB1 in response to $P$. sojae. In addition, we found that the expression levels of GmPIB1 in GmPSMD-OE transgenic soybean hairy roots and GmPSMD in GmPIB1-OE transgenic soybean hairy roots increased, respectively (Figures $\mathbf{8 B}, \mathbf{C}$ ), which proved that they were a pair of positive regulatory factors. In this study, both GmPIB1 and GmPSMD can improve the resistance of soybean hairy roots to $P$. sojae by decreasing the production of ROS. The difference is that GmPIB1 inhibits the expression of GmSPOD1, while GmPSMD influences the activity of antioxidant enzymes and inhibit the accumulation of ROS. Taken together, these data indicate that GmPSMD could improve resistance to $P$. sojae in 
soybean by improving antioxidant enzymes activity of CAT, POD, SOD, and GPX to reduce levels of ROS.

\section{DATA AVAILABILITY STATEMENT}

All datasets generated for this study are included in the article/Supplementary Material.

\section{AUTHOR CONTRIBUTIONS}

PX and SZ designed the experiments. TL, HW, ZL, and ZP performed the experiments. CZ, MZ, BN, BS, SL, ZH, WW, YL, and JW analyzed the data. TL, PX, and SZ wrote the manuscript. All authors contributed to the article and approved the submitted version.

\section{FUNDING}

This work was supported by the NSFC Projects (31671719 and 31971972), the Natural Science Foundation

\section{REFERENCES}

Azevedo, C., Sadanandom, A., Kitagawa, K., Freialdenhoven, A., Shirasu, K., and Schulze-Lefert, P. (2002). The Rar1 interactor Sgt1, an essential component of gene-triggered disease resistance. Science 295, 2073-2076. doi: 10.1126/science. 1067554

Bhattacharyya, S., Yu, H., Mim, C., and Matouschek, A. (2014). Regulated protein turnover snapshots of the proteasome in action. Nat. Rev. Mol. Cell Biol. 15, 122-133. doi: 10.1038/nrm3741

Boyes, D. C., Nam, J., and Dangl, J. L. (1998). The Arabidopsis thaliana Rpm1 disease resistance gene product is a peripheral plasma membrane protein that is degraded coincident with the hypersensitive response. Proc. Natl. Acad. Sci. U.S.A. 95, 15849-15854. doi: 10.1073/pnas.95.26.15849

Cao, Y., Zhang, Z. W., Xue, L. W., Du, J. B., Shang, J., Xu, F., et al. (2009). Lack of salicylic acid in Arabidopsis protects plants against moderate salt stress. Z. Naturforsch. C 64, 231-238. doi: 10.1515/znc-2009-3-414

Cheng, Q., Dong, L., Gao, T., Liu, T., Li, N., Wang, L., et al. (2018). The bHLH Transcription factor GmPIB1 facilitates resistance to Phytophthora sojae in Glycine max. J. Exp. Bot. 69, 2527-2541. doi: 10.1093/jxb/ery103

Cheng, Q., Li, N. H., Dong, L. D., Zhang, D. Y., Fan, S. J., Jiang, L. Y., et al. (2015). Overexpression of soybean isoflavone reductase (GmIFR) enhances resistance to Phytophthora sojae in soybean. Front. Plant Sci. 6:1024. doi: 10.3389/fpls. 2015.01024

Clough, R. C., Jordan-Beebe, E. T., Lohman, K. N., Marita, J. M., and Vierstra, R. D. (1999). Sequences within both the N-and C-terminal domains of phytochrome A are required for PFR ubiquitination and degradation. Plant J. 17, 155-167. doi: 10.1046/j.1365-313X.1999.00360.x

Coll, N. S., Epple, P., and Dangl, J. L. (2011). Programmed cell death in the plant immune system. Cell Death Differ. 18, 1247-1256. doi: 10.1038/cdd.2011.37

Dill, A. (2004). The Arabidopsis F-box protein sleepyi targets gibberellin signaling repressors for gibberellin-induced degradation. Plant Cell 16, 1392-1405. doi: $10.1105 /$ tpc.020958

Fehr, W. R., Caviness, C. E., Burmood, D. T., and Pennington, J. S. (1971). Stage of development descriptions for soybeans, Glycine Max (L.) Merrill. Crop Sci. 11, $1-11$.

Fleet, C. M., and Sun, T. P. (2005). A dell Acate balance: the role of gibberellin in plant morphogenesis. Curr. Opin. Plant Biol. 8, 77-85.

Foyer, C. H., and Shigeoka, S. (2011). Understanding oxidative stress and antioxidant functions to enhance photosynthesis. Plant Physiol. 155, 93-100. doi: $10.1104 /$ pp.110.166181 of Heilongjiang Province (ZD2019C001), and the Outstanding Talents and Innovative Team of Agricultural Scientific Research.

\section{SUPPLEMENTARY MATERIAL}

The Supplementary Material for this article can be found online at: https://www.frontiersin.org/articles/10.3389/fpls.2021. 513388/full\#supplementary-material

Supplementary Figure 1 | (A) Construction of pCAMBIA3301-GmPIB1 recombinant vector. (B) Immunoblotting analysis of GmPIB1-OE transgenic soybean hairy roots with hairy roots carrying empty vector (EV) as control. Total protein extracts were analyzed in a 12\% SDS-PAGE gel and probed with an anti-Myc antibody. (C) Test strips for detection of GmPSMD transgenic soybean hairy roots.

Supplementary Figure 2 | Preliminary interaction verification of GmPIB1 with candidate proteins in yeast cells.

Supplementary Table 1 | Primer sequences used in this study.

Supplementary Table 2 | Candidate genes from mass spectrum data.

Hardtke, C. S., Gohda, K., Osterlund, M. T., Oyama, T., Okada, K., and Deng, X. W. (2000). Hy5 stability and activity in Arabidopsis is regulated by phosphorylation in its copl binding domain. ЕMBO J. 19, 4997-5006.

Hatsugai, N., Yamada, K., Goto-Yamada, S., and Hara-Nishimura, I. (2015). Vacuolar processing enzyme in plant programmed cell death. Front. Plant Sci. 6:234. doi: 10.3389/fpls.2015.00234

Hough, R., Pratt, G., and Rechsteiner, M. (1987). Purification of two high molecular weight proteases from rabbit reticulocyte lysate. J Biol. Chem. 262, 8303-8313.

Hückelhoven, R., and Kogel, K. H. (2003). Reactive oxygen intermediates in plant microbe interactions: who is who in powdery mildew resistance. Planta 216, 891-902. doi: 10.2307/23387696

Itoh, H., Ueguchi-Tanaka, M., Sato, Y., Ashikari, M., and Matsuoka, M. (2002). The gibberellin signaling pathway is regulated by the appearance and disappearance of slender ricel in nuclei. Plant Cell 14, 57-70. doi: 10.1105/tpc.010319

Jabs, T., Tschöpe, M., Colling, C., Hahlbrock, K., and Scheel, D. (1997). Elicitor-stimulated ion-fluxes and $\mathrm{O}_{2}{ }^{-}$from the oxidative burst are essential components in triggering defense gene activation and phytoalexin synthesis in parsley. Proc. Natl. Acad. Sci. U.S.A. 94, 4800-4805. doi: 10.1073/pnas.94.9.4800

Jones, J. D., and Dangl, J. L. (2006). The plant immune system. Nature 444, 323-329. doi: 10.1038/nature05286

Kerschen, A., Napoli, C. A., Jorgensen, R. A., and Muller, A. E. (2004). Effectiveness of RNA interference in transgenic plants. FEBS Lett. 566, 223-228. doi: 10.1016/ j.febslet.2004.04.043

Kish-Trier, E., and Hill, C. (2013). Structural biology of the proteasome. Annu. Rev. Biophys. 42, 29-49. doi: 10.1146/annurev-biophys-083012-130417

Kisselev, A. F., and Goldberg, A. L. (2005). Monitoring activity and inhibition of 26S proteasomes with fluorogenic peptide substrates. Methods Enzymol. 398, 364-378. doi: 10.1016/S0076-6879(05)98030-0

Kong, G., Zhao, Y., Jing, M., Huang, J., Yang, J., Xia, Y., et al. (2015). The activation of phytophthora effector Avr3b by plant cyclophilin is required for the nudix hydrolase activity of Avr3b. PLoS Pathog. 11:e1005139. doi: 10.1371/journal. ppat.1005139

Kurepa, J., and Smalle, J. (2008). Structure, function and regulation of plant proteasomes. Biochimie 90, 324-335. doi: 10.1016/j.biochi.2007.07.019

Liu, J., Park, C. H., He, F., Nagano, M., Wang, M., Bellizzi, M., et al. (2015). The RhoGAP SPIN6 associates with SPL11 and OsRac1 and negatively regulates programmed cell death and innate immunity in rice. PLoS Pathog. 11:1004629. doi: 10.1371/journal.ppat.1004629

Luo, H., Laluk, K., Lai, Z., Veronese, P., Song, F., and Mengiste, T. (2010). The Arabidopsis botrytis susceptible1 interactor defines a subclass of ring E3 ligases 
that regulate pathogen and stress responses. Plant Physiol. 154, 1766-1782. doi: $10.1104 /$ pp.110.163915

Marino, D., Peeters, N., and Rivas, S. (2012). Ubiquitination during plant immune signaling. Plant Physiol. 160, 15-27. doi: 10.2307/23274670

Mittler, R., Vanderauwera, S., Gollery, M., and Van Breusegem, F. (2004). Reactive oxygen gene network of plants. Trends Plant Sci. 9, 490-498. doi: 10.1016/j. tplants.2004.08.009

Noctor, G., and Foyer, C. H. (1998). Ascorbate and glutathione: keeping active oxygen under control. Annu. Rev. of Plant Physiol. Plant Mol. Biol. 49, 249-279. doi: 10.1146/annurev.arplant.49.1.249

Park, C. H., Chen, S., Shirsekar, G., Zhou, B., Khang, C. H., Songkumarn, P., et al. (2012). The magnapor the oryzae effector AvrPiz-t targets the RING E3 ubiquitin ligase APIP6 to suppress pathogen-associated molecular pat-terntriggered immunity in rice. Plant Cell 24, 4748-4762. doi: 10.1105/tpc.112. 105429

Paz, M. M., Shou, H., Guo, Z., Zhang, Z., Banerjee, A. K., and Wang, K. (2004). Assessment of conditions affecting Agrobacterium-mediated soybean transformation using the cotyledonary node explant. Euphytica 136, 167-179. doi: 10.1023/B:EUPH.0000030669.75809.dc

Potuschak, T., Lechner, E., Parmentier, Y., Yanagisawa, S., Grava, S., and Koncz, C. (2003). EIN3-dependent regulation of plant ethylene hormone signaling by two Arabidopsis F box proteins: EBF1 and EBF2. Cell 115, 679-689. doi: 10.1016/S0092-8674(03)00968-1

Qian, H. F., Chen, W., Sun, L. W., Jin, Y. X., Liu, W. P., and Fu, Z. W. (2009). Inhibitory effects of paraquat on photosynthesis and the response to oxidative stress in Chlorella vulgaris. Ecotoxicology 18, 537-543. doi: 10.1007/s10646-0090311-8

Qiao, H., Wang, H., Zhao, L., Zhou, J., Huang, J., and Zhang, Y. (2004). The F-box protein ahslf-s2 physically interacts with s-rnases that may be inhibited by the ubiquitin/26S proteasome pathway of protein degradation during compatible pollination in antirrhinum. Plant Cell 16, 582-595. doi: 10.1105/tpc.017673

Schmitthenner, A. F. (1972). Evidence for a new race of Phytophthora megasperma var. sojae pathogenic to soybean. Plant Dis. 56, 536-539. doi: 10.1094/Phyto70-977

Schmitthenner, A. F. (1985). Problems and progress in control of Phytophthora root rot of soybean. Plant Dis. 69, 362-368. doi: 10.1094/PD-69-362

Schmitthenner, A. F., Lohnes, D. G., and Nickell, C. D. (1996). Origin of soybean alleles for Phytophthora resistance in China. Crop Sci. 36, 1689-1692. doi: 10.2135/cropsci1996.0011183X003600060045x

Schultz, T. F. (2001). A role for LKP2 in the circadian clock of Arabidopsis. Plant Cell 13, 2659-2670. doi: 10.1105/tpc.13.12.2659

Schwechheimer, C., and Deng, X. W. (2001). COP9 signalosome revisited: a novel mediator of protein degradation. Trends Cell Biol. 11, 420-426. doi: 10.1016/ S0962-8924(01)02091-8

Shigeoka, S., and Maruta, T. (2014). Cellular redox regulation signaling, and stress response in plants. Biosci. Biotechnol. Biochem. 78, 1457-1470. doi: 10.1080/ 09168451.2014 .942254

Silverstone, A. L., Jung, H. S., and Dill, A. (2001). Repressing a repressor: gibberellin-induced rapid reduction of the RGA protein in Arabidopsis. Plant Cell 13, 1555-1566. doi: 10.1105/tpc.13.7.1555

Smalle, J., Kurepa, J., Yang, P., Thomas, J., Babiychuk, E., and Richard, D. (2003). The pleiotropic role of the 26S proteasome subunit RPN10 in Arabidopsis growth and development supports asubstrate-specific function in abscisic acid signaling. Plant Cell 15, 965-980. doi: 10.1105/tpc.009217

Smalle, J., and Vierstra, R. D. (2004). The ubiquitin 26S proteasome proteolytic pathway. Annu. Rev. Plant Biol. 55, 555-590. doi: 10.1146/annurev.arplant.55. 031903

Stadtmueller, B., Hill, C., and Förster, A. (2011). Proteasome activators. Mol. Cell 41, 8-19. doi: 10.1016/j.molcel.2010.12.020

Suzuki, N., Miller, G., Morales, J., Shulaev, V., Torres, M. A., and Mittler, R. (2011). Respiratory burst oxidases: the engines of ROS signaling. Curr. Opin. Plant Biol. 14, 691-699. doi: 10.1016/j.pbi.2011.07.014

Tanaka, K., Ii, K., Ichihara, A., Waxman, L., and Goldberg, A. L. (1986). A high molecular weight protease in the cytosol of rat liver purification, enzymological properties and tissue distribution. J. Biol. Chem. 261, 15197-15203.
Tyler, B. M. (2007). Phytophthora sojae: root rot pathogen of soybean and model oomycete. Mol. Plant Pathol. 8, 1-8.

Tyler, L. (2004). DELLA proteins and gibberellin-regulated seed germination and floral development in Arabidopsis. Plant Physiol. 135, 1008-1019. doi: 10.1104/ pp.104.039578

Üstün, S., Bartetzko, V., and Börnke, F. (2013). The Xanthomonas campestris type III effector XopJ targets the host cell proteasome to suppress salicylicacid mediated plant defence. PLoS Pathog. 9:1003427. doi: 10.1371/journal.ppat. 1003427

Üstün, S., and Börnke, F. (2015). The Xanthomonas campestris type III effector XopJ proteolytically degrades proteasome subunit RPT6. Plant Physiol. 168, 107-119. doi: 10.1104/pp.15.00132

Üstün, S., König, P., Guttman, D. S., and Börnke, F. (2014). HopZ4 from Pseudomonas syringae, a member of the HopZ type III effector family from the YopJ superfamily, inhibits the proteasome in plants. Mol. Plant Microbe Interact. 27, 611-623. doi: 10.1094/MPMI-12-13-0363-R

Velikova, V., Yordanov, I., and Edreva, A. (2000). Oxidative stress and some antioxidant systems in acid rain-treated bean plants: protective role of exogenous polyamines. Plant Sci. 151, 59-66. doi: 10.1016/s0168-9452(99) 00197-1

Ward, E., Lazarovits, G., Unwin, C., and BuzzellR, I. (1979). Hypocotyl reactions and glyceollin in soybeans inoculated with zoospores of Phytophthora megaspuma var. sojae. Phytopathology 69, 951-955. doi: 10.1094/Phyto69-951

Wu, G., Shortt, B. J., Lawrence, E. B., Leôn, J., Fitzsimmons, K. C., and Shah, D. M. (1997). Activation of host defense mechanisms by elevated production of $\mathrm{H}_{2} \mathrm{O}_{2}$ in transgenic plants. Plant Physiol. 115, 427-435. doi: 10.1104/pp.115.2.419

Xu, P., Jiang, L., Wu, J., Li, W., Fan, S., and Zhang, S. (2014). Isolation and characterization of a pathogenesis-related protein 10 gene (GmPR10) with induced expression in soybean (Glycine max) during infection with Phytophthora sojae. Mol. Biol. Rep. 41, 4899-4909. doi: 10.1007/s11033-0143356-6

Yang, X., Ruff, R., Meng, X., and Workneh, F. (1996). Races of Phytophthora sojae in Iowa soybean fields. Plant Dis. 80, 1418-1420. doi: 10.1094/PD-80-1418

Yu, D., Yu, F., Du, C., Li, X., Zhao, X., and Liu, X. (2015). RPN1a, a subunit of the 26S proteasome, controls trichome development in Arabidopsis. Plant Physiol. Biochem. 88, 82-88. doi: 10.1016/j.plaphy.2015.01.012

Zhang, C., Wang, X., Zhang, F., Dong, L., Wu, J., Cheng, Q., et al. (2017). Phenylalanine ammonia-lyase2.1 contributes to the soybean response towards Phytophthora sojae infection. Sci. Rep. 7:7242. doi: 10.1038/s41598-017-07 832-2

Zhang, H., Huang, L., Dai, Y., Liu, S., Hong, Y., Tian, L., et al. (2015). Arabidopsis AtERF15 positively regulates immunity against Pseudomonas syringae pv. tomato DC3000 and Botrytis cinerea. Front. Plant Sci. 6:686. doi: 10.3389/fpls. 2015.00686

Zhang, H. J., Hong, Y. B., Huang, L., Li, D. Y., and Song, F. M. (2016). Arabidopsis AtERF014 acts as a dual regulator that differentially modulates immunity against Pseudomonas syringae pv. tomato and Botrytis cinerea. Sci. Rep. 6:30251. doi: $10.1038 /$ srep30251

Zhang, R., Chae, S., Kang, K. A., Piao, M. J., Ko, D. O., Wang, Z. H., et al. (2008). Protective effect of butin against hydrogen peroxide-induced apoptosis by scavenging reactive oxygen species and activating antioxidant enzymes. Mol. Cell. Biochem. 318, 33-42. doi: 10.1007/s11010-008-9854-x

Conflict of Interest: The authors declare that the research was conducted in the absence of any commercial or financial relationships that could be construed as a potential conflict of interest.

Copyright (C) 2021 Liu, Wang, Liu, Pang, Zhang, Zhao, Ning, Song, Liu, He, Wei, $W u, L i u, X u$ and Zhang. This is an open-access article distributed under the terms of the Creative Commons Attribution License (CC BY). The use, distribution or reproduction in other forums is permitted, provided the original author(s) and the copyright owner(s) are credited and that the original publication in this journal is cited, in accordance with accepted academic practice. No use, distribution or reproduction is permitted which does not comply with these terms. 